\title{
A new ex-ante efficiency criterion and implications for the probabilistic serial mechanism ${ }^{\text {in }}$
}

\author{
Battal Doğan ${ }^{\mathrm{a}}$, Serhat Doğan ${ }^{\mathrm{b}}$, Kemal Y1ldız ${ }^{\mathrm{b}, *}$ \\ a Faculty of Business and Economics, University of Lausanne, Switzerland \\ ${ }^{\mathrm{b}}$ Department of Economics, Bilkent University, Turkey
}

Received 18 May 2016; final version received 10 January 2018; accepted 22 January 2018

Available online 4 February 2018

\begin{abstract}
We introduce and analyze an efficiency criterion for probabilistic assignment of objects, when only ordinal preference information is available. This efficiency criterion is based on the following domination relation: a probabilistic assignment dominates another assignment if it is ex-ante efficient for a strictly larger set of utility profiles consistent with the ordinal preferences. We provide a simple characterization of this domination relation. We revisit an extensively studied assignment mechanism, the Probabilistic Serial mechanism (Bogomolnaia and Moulin, 2001), which always chooses a "fair" assignment. We show that the Probabilistic Serial assignment may be dominated by another fair assignment. We provide conditions under which the serial assignment is undominated among fair assignments.
\end{abstract}

(c) 2018 Elsevier Inc. All rights reserved.

JEL classification: C60; C71; C78; D61

Keywords: Ex-ante efficiency; Probabilistic assignment; Fairness; Probabilistic serial mechanism

\footnotetext{
to We are grateful to Paulo Barelli, Srihari Govindan, Bettina Klaus, Efe Ok, Ariel Rubinstein, William Thomson, seminar participants at Bilkent University and the Bosphorus Workshop on Economic Design, and the associate editor and three anonymous referees for their helpful comments. Battal Doğan gratefully acknowledges the support from the Swiss National Science Foundation (SNSF) and Kemal Yıldız gratefully acknowledges the support from the Scientific and Research Council of Turkey (TÜBITAK) under grant number 115K549.

* Corresponding author.

E-mail addresses: battaldogan@gmail.com (B. Doğan), dserhat@bilkent.edu.tr (S. Doğan), kemal.yildiz@bilkent.edu.tr (K. Yıldız).
} 


\section{Introduction}

We study the assignment problem in which $n$ objects are to be allocated among $n$ agents such that each agent receives an object and monetary compensations are not possible. Applications include assigning houses to agents or students to schools. Motivated by fairness concerns, probabilistic assignments (lotteries over sure assignments) have been extensively studied in the literature.

Starting with the seminal study by Hylland and Zeckhauser (1979), the vast majority of the literature assumes that each agent derives a utility for being assigned an object, and his ex-ante evaluation of a probabilistic assignment is his expected utility for that probabilistic assignment. In other words, agents are endowed with von-Neumann-Morgenstern (vNM) preferences over probabilistic assignments. In this setup, a natural efficiency requirement for a probabilistic assignment is ex-ante efficiency: the probabilistic assignment maximizes the sum of the expected utilities. Obviously, evaluating the ex-ante efficiency of a probabilistic assignment requires knowledge of the vNM preferences. However, ordinal allocation mechanisms that elicit only preferences over sure objects have been particularly studied in the literature. ${ }^{1}$ When an ordinal mechanism is used, agents are asked to report their preference orderings over objects. ${ }^{2}$ Therefore, the efficiency of an assignment has to be evaluated based only on the ordinal preference information. In this ordinal environment, we propose the following efficiency criterion: a probabilistic assignment dominates another assignment in social welfare terms, or sw-dominates, if

i. whenever the latter assignment is ex-ante efficient at a utility profile consistent with the ordinal preferences, the former assignment is ex-ante efficient too; and

ii. there is a utility profile consistent with the ordinal preferences at which the latter assignment is not ex-ante efficient but the former assignment is ex-ante efficient.

Although our dominance notion is based on all possible utility representations of the ordinal preferences, it does not require any knowledge of the particular utilities. We call an assignment sw-efficient if it is not dominated in social welfare terms.

The common method in the literature to ordinally evaluate the efficiency of a probabilistic assignment is based on first order stochastic dominance. This efficiency notion, introduced by Bogomolnaia and Moulin (2001), is called sd-efficiency: a probabilistic assignment is sd-efficient if it is not stochastically dominated by any other assignment. ${ }^{3}$ McLennan (2002) shows that an assignment is sd-efficient if and only if there is a utility profile at which it is ex-ante efficient, which readily implies that any sd-efficient assignment sw-dominates any assignment that is not sd-efficient. Put differently, sw-domination provides a refinement of sd-domination that can facilitate the comparison of probabilistic assignments among which there is no stochastic dominance relationship.

As for the applications, the indeterminacy due to stochastic dominance might make it difficult to compare probabilistic assignment mechanisms. For example, Pathak (2008) compares the

\footnotetext{
1 See Bogomolnaia and Moulin (2001) for several justifications for observing ordinal mechanisms in practice.

2 Part of the literature focuses on strict preferences such that each agent reports a complete, transitive, and antisymmetric ordering over objects. Unless otherwise noted, we allow for weak preferences that are not necessarily anti-symmetric.

3 Bogomolnaia and Moulin (2001) refers to sd-efficiency as "ordinal efficiency." Here, we use the terminology of Thomson (2010).
} 
performance of probabilistic serial mechanism to the random serial dictatorship mechanism by using the data of student placement in public schools in New York City. For $44 \%$ of the students the probabilistic assignments generated by the two mechanisms are not comparable with respect to first order stochastic dominance. Here, we show that sw-domination induces a clean ranking of sd-efficient assignments, which enables us to compare the well-known assignment mechanisms such as random serial dictatorship and probabilistic serial assignment mechanisms.

We show that if preferences are strict (no agent is indifferent between two different objects), an sd-efficient assignment $\pi$ sw-dominates another sd-efficient assignment $\pi^{\prime}$ if and only if $\pi$ has a smaller support, i.e. the set of agent-object pairs assigned with positive probability in $\pi$ is a proper subset of the set of agent-object pairs assigned with positive probability in $\pi^{\prime}$. If preferences are weak (indifference is allowed), we extend the support of an assignment so that it possibly includes an agent-object pair that is not assigned with positive probability, provided that there is an "equivalent assignment" that includes the pair in its support. Then, we show that an sd-efficient assignment $\pi$ sw-dominates another sd-efficient assignment $\pi^{\prime}$ if and only if $\pi$ has a smaller extended support. A consequence of these results is that when preferences are strict, the only sw-efficient assignments are the Pareto efficient deterministic assignments; and when preferences are weak, the only undominated assignments are the sd-efficient assignments in which each agent is indifferent among the objects that he is assigned with positive probability.

Our analysis shows that each sw-efficient assignment is essentially deterministic. Given that the main motivation for probabilistic assignments is fairness, this observation indicates a tradeoff between fairness and efficiency and provides an insight about the difficulty of achieving efficiency together with fairness in the probabilistic setting. Another takeaway is that, in a setting where randomization is required to establish fairness, the best policy in terms of (social welfare) efficiency is to establish fairness with a minimum amount of randomization.

The proof of Theorem 1 builds upon a result by McLennan (2002) and its constructive proof by Manea (2008), which together show that for each sd-efficient assignment, a utility profile consistent with the ordinal preferences can be constructed at which the assignment is ex-ante efficient. Here, we are able to describe the general structure of the set of utility profiles at which a given assignment is ex-ante efficient. ${ }^{4}$

In the second part of the paper, we revisit an extensively studied probabilistic assignment mechanism, namely the Probabilistic Serial (PS) mechanism. Bogomolnaia and Moulin (2001) introduce the $P S$ mechanism and show that it always chooses a fair and sd-efficient assignment. ${ }^{5}$ We observe that, without sacrificing fairness, the $P S$ mechanism can be improved in sw-efficiency. Given this observation, an important question is "When is it possible to have a fair assignment that sw-dominates the serial assignment?". To answer this question, we consider a directed graph, the configuration of which depends on the given ordinal preference profile. We show that a special connectedness property of this graph plays a critical role in understanding at which preference profiles the serial assignment is sw-efficient among fair assignments.

\footnotetext{
4 For example, it follows from the proof of Theorem 1 that for two sd-efficient probabilistic assignments the set of utility profiles at which each assignment is ex-ante efficient are the same if and only if the agent-object pairs assigned with positive probability in these two assignments are the same.

5 See Section 4 for the formal definition of fairness.
} 


\section{The framework}

Let $N$ be a set of $n$ agents and $A$ be a set of $n$ objects. For each $i \in N$, the preference relation of $i$, which we denote by $R_{i}$, is a weak order on $A$, i.e. it is transitive and complete. Given a pair of objects $a, b \in A$, we write $a P_{i} b$ when $a R_{i} b$ but not $b R_{i} a$; we write $a I_{i} b$ when $a R_{i} b$ and $b R_{i} a$, and call $I_{i}$ the associated indifference relation. Let $\mathcal{R}_{i}$ denote the set of all possible preference relations for $i$, and $\mathcal{R} \equiv \times_{i \in N} \mathcal{R}_{i}$ denote the set of all possible preference profiles, which we also call the weak preference domain. Let $\mathcal{R}_{i}^{S} \subset \mathcal{R}_{i}$ denote the set of all possible strict preference relations for $i$, i.e. the set of all anti-symmetric preference relations in $\mathcal{R}_{i}$, and $\mathcal{R}^{S} \equiv \times_{i \in N} \mathcal{R}_{i}^{S}$ denote the set of all possible strict preference profiles, which we also call the strict preference domain. ${ }^{6}$ Note that for each $R_{i} \in \mathcal{R}_{i}^{S}$ and each pair of objects $a, b \in A, a I_{i} b$ implies that $a=b$.

A deterministic assignment is a one-to-one function from $N$ to $A$. A deterministic assignment can be represented by an $n \times n$ matrix with rows indexed by agents and columns indexed by objects, and having entries in $\{0,1\}$ such that each row and each column has exactly one 1 . Such a matrix is called a permutation matrix. For each $(i, a) \in N \times A$, having 1 in the $(i, a)$ entry indicates that $i$ is assigned $a$. A probabilistic assignment (an assignment hereafter) is a probability distribution over deterministic assignments. An assignment can be represented by an $n \times n$ matrix having entries in $[0,1]$ such that the sum of the entries in each row and each column is 1 . Such a matrix is called a doubly stochastic matrix. For each assignment $\pi$, and each pair $(i, a) \in N \times A$, the entry $\pi_{i a}$, which we also write as $\pi_{i}(a)$ or $\pi(i, a)$, indicates the probability that $i$ is assigned to $a$ at $\pi$. Since each doubly stochastic matrix can be represented as a convex combination of permutation matrices (Birkhoff, 1946 and Von Neumann, 1953), the set of all doubly stochastic matrices is the set of all assignments. Let $\Pi$ be the set of all doubly stochastic matrices.

We denote the collection of all lotteries over $A$ by $\mathcal{L}(A)$. For each $i \in N$, a von-NeumannMorgenstern (vNM) utility function $u_{i}$ is a real valued mapping on $A$, i.e. $u_{i}: A \rightarrow \mathbb{R}$. For each $i \in N$ with preferences $R_{i} \in \mathcal{R}_{i}$, a vNM utility function $u_{i}$ is consistent with $R_{i}$ if for each pair $(a, b) \in A$, we have $u_{i}(a) \geq u_{i}(b)$ if and only if $a R_{i} b$. We obtain the corresponding preferences of $i$ over $\mathcal{L}(A)$ by comparing the expected utilities, where the expected utility from $\pi_{i} \in \mathcal{L}(A)$ is $\sum_{a \in A} \pi_{i}(a) u_{i}(a)$.

Next, we define the sd-efficiency of an assignment. The formulation of sd-efficiency is independent of any vNM utility specification consistent with the ordinal preferences. Let $\pi, \pi^{\prime} \in \Pi$, $i \in N$, and $R \in \mathcal{R}$. We say that $\pi_{i}$ stochastically dominates $\pi_{i}^{\prime}$ at $R_{i}$, or simply $\pi_{i}$ sd-dominates $\pi_{i}^{\prime}$ at $R_{i}$, if for each $a \in A$,

$$
\sum_{b: b R_{i} a} \pi_{i}(b) \geq \sum_{b: b R_{i} a} \pi_{i}^{\prime}(b) .
$$

We say that $\pi$ stochastically dominates $\pi^{\prime}$ at $R$, or simply $\pi$ sd-dominates $\pi^{\prime}$ at $R$, if $\pi \neq \pi^{\prime}$ and for each $i \in N, \pi_{i}$ sd-dominates $\pi_{i}^{\prime}$ at $R_{i}$. An assignment $\pi \in \Pi$ is sd-efficient at $R$ if no assignment sd-dominates $\pi$ at $R$. Let $P^{s d}(R)$ denote the set of sd-efficient assignments at $R$.

\footnotetext{
6 Since we allow our agents to be indifferent among objects, multi-unit assignment problems, in which each object may have multiple copies, turn out to be a special case of our framework. In that we can consider preference profiles such that each agent is indifferent between different copies of the same object.
} 


\section{SW-domination and a characterization}

For each utility profile $u=\left(u_{i}(.)\right)_{i \in N}$ and assignment $\pi$, the ex-ante social welfare at $(u, \pi)$ is the sum of the expected utilities of the agents, that is:

$$
S W(u, \pi)=\sum_{(i, a) \in N \times A} \pi_{i}(a) u_{i}(a) .
$$

An assignment $\pi$ is ex-ante efficient at a utility profile $u$ if it maximizes the social welfare at $u$, i.e. $\pi \in \arg \max _{\pi^{\prime} \in \Pi} S W\left(u, \pi^{\prime}\right)$.

Let $\pi, \pi^{\prime} \in \Pi$ and $R \in \mathcal{R}$. An assignment $\pi$ dominates $\pi^{\prime}$ in social welfare terms at $R$, or simply $\pi$ sw-dominates $\pi^{\prime}$ at $R$ if

i. for each utility profile $u$ consistent with $R$, if $\pi^{\prime}$ is ex-ante efficient at $u$, then $\pi$ is ex-ante efficient at $u$ too, and

ii. there is a utility profile $u$ consistent with $R$ at which $\pi$ is ex-ante efficient but $\pi^{\prime}$ is not ex-ante efficient.

An assignment $\pi$ is sw-efficient at $R$ if there is no assignment $\pi^{\prime}$ that sw-dominates $\pi$ at $R$. For each $\pi, \pi^{\prime} \in \Pi, \pi$ and $\pi^{\prime}$ are equivalent in social welfare terms at $R$, or simply $\pi$ and $\pi^{\prime}$ are sw-equivalent at $R$ if for each utility profile $u$ consistent with $R, \pi$ is ex-ante efficient at $u$ if and only if $\pi^{\prime}$ is ex-ante efficient at $u$. An assignment $\pi$ weakly dominates $\pi^{\prime}$ in social welfare terms at $R$, or simply $\pi$ weakly sw-dominates $\pi^{\prime}$ at $R$ if $\pi$ sw-dominates $\pi^{\prime}$ or $\pi$ and $\pi^{\prime}$ are sw-equivalent at $R$. An assignment $\pi$ is strongly sw-efficient at $R$ if there is no assignment $\pi^{\prime}$ that weakly sw-dominates $\pi$ at $R$. McLennan (2002) shows that an assignment is sd-efficient if and only if there is a utility profile at which it is ex-ante efficient, which readily implies that any sd-efficient assignment sw-dominates any assignment that is not sd-efficient. Thus, sw-domination provides a refinement of sd-domination.

Although the sw-domination notion is based on all possible utility representations of the preferences, it does not require any knowledge of the particular utilities. Hence, knowing ordinal preferences suffice for the comparison. However, since there would be a huge collection of utility profiles consistent with any given ordinal preference profile, this comparison can be computationally burdensome. Therefore, it may not be clear which assignments are sw-efficient. In what follows, we provide a characterization of sw-domination. From the characterization, it follows that sw-efficiency implies sd-efficiency. Moreover, a simple relation among sd-efficient assignments identifies whether one of these assignments sw-dominates the other.

First, we introduce some notation. For each $\pi \in \Pi$, we refer to the collection of pairs $(i, a) \in$ $N \times A$ with $\pi_{i}(a)>0$ as the support of $\pi$, denoted by $\operatorname{Sp}(\pi)$. For each $\pi, \pi^{\prime} \in \Pi, S p(\pi) \subsetneq$ $S p\left(\pi^{\prime}\right)$ means that for each pair $(i, a) \in N \times A$, if $\pi_{i}(a)>0$ then $\pi_{i}^{\prime}(a)>0$, and there is a pair $(i, a) \in N \times A$ such that $\pi_{i}(a)=0$, but $\pi_{i}^{\prime}(a)>0$.

The support notion will be critical in characterizing sw-domination on the strict preference domain. For a characterization on the weak preference domain, an extension of the support notion will be the key. The following relation on $(\pi, R)$, denoted by $\sim_{(\pi, R)}$, will be helpful to define the extension. For each $(i, a),(j, b) \in N \times A,(i, a) \sim_{(\pi, R)}(j, b)$ if and only if $\pi_{i}(b)>0$ and $a I_{i} b$.

Note that if $(i, a) \sim_{(\pi, R)}(j, b)$, then for each $k \in N,(i, a) \sim_{(\pi, R)}(k, b)$. A cycle at $\sim_{(\pi, R)}$ is a sequence of pairs (not necessarily distinct) $\left(i_{1}, a_{1}\right),\left(i_{2}, a_{2}\right), \ldots,\left(i_{k}, a_{k}\right) \in N \times A$ such that $\left(i_{1}, a_{1}\right) \sim_{(\pi, R)}\left(i_{2}, a_{2}\right) \sim_{(\pi, R)} \ldots \sim_{(\pi, R)}\left(i_{k}, a_{k}\right) \sim_{(\pi, R)}\left(i_{1}, a_{1}\right)$. 
Let $R$ be a preference profile and $\pi$ be an assignment. A pair $(i, a) \in N \times A$ is in the extended support of $\pi$ relative to $R$, denoted by $(i, a) \in \operatorname{Ext} \operatorname{Sp}(\pi, R)$, if there is a cycle of $\sim_{(\pi, R)}$ that contains $(i, a)$. To get some intuition, imagine that $(i, a) \notin S p(\pi)$ and we trade a small probability along the cycle such that $i_{1}$ gets less of $a_{2}$ and more of $a_{1}, i_{2}$ gets less of $a_{3}$ and more of $a_{2}$, and so on. Note that in the new assignment, each agent's expected utility is the same as before; moreover, $(i, a)$ is now included in the support. In a sense, although $(i, a)$ is not included in the support $\pi$, it is included in the support of an equivalent assignment. Also observe that, in order to have $(i, a) \in \operatorname{Ext} \operatorname{Sp}(\pi, R) \backslash S p(\pi)$, there must be an object $b \in A$ such that $a I_{i} b, \pi_{i}(b)>0$. The following example illustrates the support and the extended support of an assignment.

Example 1. Let $N=\{1,2,3\}$ and $A=\{a, b, c\}$. Let $R \in \mathcal{R}$ and $\pi \in \Pi$ be as depicted in Fig. 1 . Note, for instance, that agent 1 is indifferent between $a$ and $b$, he prefers $a$ or $b$ to $c$, and he is assigned $b$ for sure at assignment $\pi$.

\begin{tabular}{|c|c|c|c|c|c|c|}
\hline$R_{1}$ & $R_{2}$ & $R_{3}$ & $\pi$ & $a$ & $b$ & $c$ \\
\hline$a, b$ & $a$ & $a, c$ & 1 & 0 & 1 & 0 \\
\hline$c$ & $b, c$ & $b$ & 2 & 0.4 & 0 & 0.6 \\
\hline & & & 3 & 0.6 & 0 & 0.4 \\
\hline
\end{tabular}

Fig. 1. The extended support of $\pi$ is $\operatorname{ExtSp}(\pi, R)=\{(1, a),(1, b),(2, a),(2, b),(2, c),(3, a),(3, c)\}$.

Note that $S p(\pi)=\{(1, b),(2, a),(2, c),(3, a),(3, c)\}$. Observe that $(1, a) \sim_{(\pi, R)}$ $(2, b) \sim_{(\pi, R)}(3, c) \sim_{(\pi, R)}(1, a)$ is a cycle of $\sim_{(\pi, R)}$. Therefore, we have $(1, a),(2, b) \in$ $\operatorname{Ext} \operatorname{Sp}(\pi, R)$. Note that $(3, b) \notin \operatorname{Ext} \operatorname{Sp}(\pi, R)$ since there is no $b^{\prime} \in A$ such that $b I_{3} b^{\prime}, \pi_{i}\left(b^{\prime}\right)>0$. Thus, $\operatorname{ExtSp}(\pi, R)=\{(1, a),(1, b),(2, a),(2, b),(2, c),(3, a),(3, c)\}$.

Next, we present a characterization of sw-domination.

Theorem 1. For each $\pi, \pi^{\prime} \in \Pi$ and $R \in \mathcal{R}$, the assignment $\pi$ sw-dominates $\pi^{\prime}$ at $R$ if and only if

i. $\pi^{\prime} \notin P^{s d}(R)$ and $\pi \in P^{s d}(R)$, or

ii. $\pi^{\prime} \in P^{s d}(R)$ and $\operatorname{ExtSp}(\pi, R) \subsetneq \operatorname{ExtSp}\left(\pi^{\prime}, R\right)$.

Proof. See Section A.1.7

The proof of Theorem 1 essentially relies on a result by McLennan (2002) and its constructive proof by Manea (2008), which together show that for each sd-efficient assignment, a utility profile consistent with the ordinal preferences can be constructed at which the assignment is ex-ante efficient. As we show in Lemma A.1, the constructed utility profile induces a common utility function $v: A \rightarrow \mathbb{R}$ on the extended support, which plays a central role in the proof. It is also worth noting that the proof reveals new information about the general structure of the set of utility profiles at which a given assignment is ex-ante efficient. In particular, for two sd-efficient probabilistic assignments, the set of utility profiles at which each assignment is ex-ante efficient

\footnotetext{
7 As for the extension to two-sided markets, one can show that the counterpart of Theorem 1 holds for the marriage problem by using the utility profile construction in Dogan and Yildiz (2016) vis à vis the use of Manea's construction for the current result.
} 
are the same if and only if the agent-object pairs assigned with positive probability in these two assignments are the same.

As a corollary to Theorem 1, for the strict and the weak preference domains we identify all the assignments that are sw-efficient.

\section{Corollary 1.}

i. For each $R \in \mathcal{R}$, an sd-efficient assignment $\pi$ is sw-equivalent to another sd-efficient assignment $\pi^{\prime}$ at $R$ if and only if $\operatorname{Ext} \operatorname{Sp}(\pi)=\operatorname{Ext} \operatorname{Sp}\left(\pi^{\prime}\right)$.

ii. For each $R \in \mathcal{R}^{S}$, an sd-efficient assignment $\pi$ sw-dominates another sd-efficient assignment $\pi^{\prime}$ at $R$ if and only if $\operatorname{Sp}(\pi) \subsetneq S p\left(\pi^{\prime}\right)$; and $\pi$ is sw-equivalent to $\pi^{\prime}$ at $R$ if and only if $\operatorname{Sp}(\pi)=\operatorname{Sp}\left(\pi^{\prime}\right)$.

iii. For each $R \in \mathcal{R}^{S}$ an assignment $\pi$ is sw-efficient at $R$ if and only if it is a Pareto efficient deterministic assignment at $R$.

iv. For each $R \in \mathcal{R}$, an assignment $\pi$ is sw-efficient at $R$ if and only if $\pi$ is sd-efficient at $\mathcal{R}$ and each agent is indifferent between the objects he is assigned with positive probability at $\pi$.

Proof. First note that (i) directly follows from the proof of Theorem 1 in Section A.1. Similarly, (ii) directly follows from Theorem 1 and the previous item. Next we show that (iii) holds. To see this let $R$ be a strict preference profile. First for the if part, note that each deterministic Pareto efficient assignment at $R$ is sd-efficient at $R$. Then, it follows from (ii) that it is sw-efficient. Next to see the only if part, note that for any assignment $\pi$ that is not deterministic, there is a Pareto efficient deterministic assignment $\mu$ such that $\operatorname{Sp}(\mu) \subsetneq S p(\pi)$ (consider an assignment in one of the decompositions of $\pi$ ). This together with Theorem 1, imply that an assignment that is not deterministic is sw-dominated by any deterministic assignment in its decomposition. Thus, the only if part holds.

Next we show that (iv) holds. To see that the only if part holds, suppose that $\pi$ is sw-efficient at $R$. Then $\pi$ is sd-efficient. If $\pi$ is deterministic, then the claim holds. Suppose $\pi$ is not deterministic. By contradiction, suppose that there exist $i \in N$ and $a, b \in A$ such that $(i, a),(i, b) \in \operatorname{Sp}(\pi)$, but $a P_{i} b$. Next, consider a decomposition, say consisting of $\left\{\mu, \mu^{\prime}, \ldots\right\}$, of $\pi$ where $i$ is assigned $a$ at $\mu$ and $b$ at $\mu^{\prime}$. Note that $\mu$ is sd-efficient. Moreover, since $(i, b) \notin \operatorname{Ext} \operatorname{Sp}(\mu, R)$, $\operatorname{Ext} \operatorname{Sp}(\mu, R) \subsetneq \operatorname{Ext} \operatorname{Sp}(\pi, R)$. It follows that $\mu$ sw-dominates $\pi$, contradicting that $\pi$ is swefficient at $R$.

Finally to see that the if part holds, suppose that $\pi$ is sd-efficient at $R$, and each agent is indifferent between the objects he receives with positive probability at $\pi$. By contradiction, suppose there exists another assignment $\pi^{\prime}$ that sw-dominates $\pi$. It follows from Theorem 1 that $\operatorname{Ext} \operatorname{Sp}\left(\pi^{\prime}, R\right) \subsetneq \operatorname{Ext} \operatorname{Sp}(\pi, R)$. Now, recall that for each $(i, a) \in \operatorname{Ext} \operatorname{Sp}\left(\pi^{\prime}, R\right)$, there exists a pair $(i, b) \in \operatorname{Sp}\left(\pi^{\prime}, R\right)$ such that agent $i$ is indifferent between $a$ and $b$. Therefore, each agent is indifferent between the objects he receives with positive probability at $\pi$ or $\pi^{\prime}$. It follows that for each utility profile $u$ consistent with $R, S W(\pi, u)=S W\left(\pi^{\prime}, u\right)$, contradicting that $\pi^{\prime}$ swdominates $\pi$.

Since the main motivation for probabilistic assignments is fairness, Corollary 1 indicates a contrast between fairness and efficiency. Think of any setting where randomization is required to establish fairness. It follows from our result that the best policy in terms of social welfare efficiency would be to establish fairness with a minimum amount of randomization. For some problems, the contrast between sw-efficiency and fairness may be extreme, in that the only fair as- 
signments are the least sw-efficient ones from among the sd-efficient assignments. For example, when agents have the same preferences, each one of the two well-known fairness requirements for probabilistic assignments, namely sd-no-envy and equal treatment of equals, pins down a unique assignment: agents share each object equally. Note that this assignment is a least swefficient assignment of sd-efficient ones, since it has full support.

\section{SW-efficiency of the probabilistic serial mechanism}

An assignment mechanism is a function $\varphi: \mathcal{R} \rightarrow \Pi$, associating an assignment with each preference profile. On the strict preference domain, a widely studied probabilistic assignment mechanism is the probabilistic serial (PS) mechanism. At each $R \in \mathcal{R}^{S}$, the $P S$ assignment is computed by the following algorithm. Consider each object as an infinitely divisible good with a one unit supply that will be eaten by agents in the time interval $[0,1]$ through the following steps:

Step 1: Each agent eats from his most preferred object. Agents eat at the same speed. When an object is completely eaten, proceed to the next step.

Steps $s \geq 2$ : Each agent eats from his most preferred object from among the ones that have not yet been completely eaten. Agents eat at the same speed. When an object is completely eaten, proceed to the next step.

The algorithm terminates when all the objects are exhausted (or equivalently when each agent has eaten in total exactly one unit of objects), and the probability that an agent receives an object in the $P S$ assignment is defined as the amount of the object the agent has eaten. We denote the $P S$ assignment at $R$ by $\pi^{p s}(R)$.

Given $R \in \mathcal{R}^{S}, a \in A$, and $t \in[0,1]$, we say that $a$ is exhausted at time $t$ in the $P S$ algorithm at $R$ if at the end of the step that ends when $a$ is completely eaten, each agent has eaten in total $t$ units of the objects. Note that for each pair $a, b \in A$, if $a$ and $b$ are exhausted at different times in the PS algorithm at $R$, then for each $i, j \in N$ with $\pi^{p s}(i, a)>0$ and $\pi^{p s}(j, b)>0$, we have $\pi^{p s}\left(i,\left\{c \in A: c R_{i} a\right\}\right) \neq \pi^{p s}\left(j,\left\{c \in A: c R_{j} b\right\}\right)$.

Bogomolnaia and Moulin (2001) show that the PS mechanism chooses an sd-efficient assignment at each strict preference profile. Another well-known probabilistic assignment mechanism is the random serial dictatorship (RD) mechanism, which draws at random an ordering of the agents from the uniform distribution, then lets them choose successively their best remaining object (the first agent in the ordering is assigned to his best object, the second agent to his best among the remaining objects, and so on). Bogomolnaia and Moulin (2001) show that $R D$ mechanism does not always choose an sd-efficient assignment. Manea (2009) shows that the inefficiency of the $R D$ mechanism prevails even in the large markets, since the probability that the resulting assignment is sd-efficient converges to zero as the number of object types becomes large. However, as Bogomolnaia and Moulin (2001) shows, there are preference profiles at which $R D$ and $P S$ mechanisms choose different assignments such that neither sd-dominates the other. Empirical observations, by Pathak (2008), in the context of school choice problem, indicate that this indeterminacy arises so often that comparing $R D$ and $P S$ mechanisms based on sd-dominance becomes difficult.

In contrast to sd-domination, it follows from our Corollary 1 that at each preference profile the $P S$ assignment weakly sw-dominates the $R D$ assignment. To see this, consider any $R \in \mathcal{R}^{S}$, if the $R D$ assignment is not sd-efficient at $R$, then the $P S$ assignment sw-dominates the $R D$ assignment. Suppose that both assignments are sd-efficient at $R$. Since the $R D$ assignment chooses each Pareto efficient deterministic assignment with a positive probability, the $R D$ assignment has the 
largest support among the ex-post efficient assignments. Then, each agent-object pair that is assigned with a positive probability in the $P S$ assignment at $R$ is also assigned with a positive probability in the $R D$ assignment at $R$. Hence, the $P S$ assignment either sw-dominates or is sw-equivalent to the $R D$ assignment at $R$. More generally, it follows that any ex-post efficient assignment weakly sw-dominates the $R D$ assignment at any preference profile.

Besides sd-efficiency, the PS mechanism also satisfies sd-envy-freeness (Bogomolnaia and Moulin, 2001), which has been a central fairness requirement in the probabilistic assignment literature: an assignment $\pi$ is sd-envy-free at $R$ if for each pair of agents $i, j \in N, \pi_{i}$ sd-dominates $\pi_{j}$ at $R_{i}$. However, it follows from Corollary 1 that the $P S$ mechanism is not sw-efficient, since there are preference profiles at which $P S$ mechanism does not choose a deterministic assignment. One natural question is the following: Given $R \in \mathcal{R}^{S}$, is the $P S$ assignment sw-efficient in the class of sd-envy-free assignments at $R$ ? Our next example shows that there is a strict preference profile for which there is an sd-envy-free assignment that sw-dominates the PS assignment and is not sw-dominated by any other sd-envy-free assignment.

Example 2. Let $N=\{1,2,3\}$ and $A=\{a, b, c\}$. Consider the following preference profile (Fig. 2).

\begin{tabular}{ccc}
$R_{1}$ & $R_{2}$ & $R_{3}$ \\
\hline$a$ & $a$ & $b$ \\
$b$ & $c$ & $c$ \\
$c$ & $b$ & $a$
\end{tabular}

\begin{tabular}{c|ccc}
$\pi^{p s}(R)$ & $a$ & $b$ & $c$ \\
\hline 1 & $\frac{1}{2}$ & $\frac{1}{4}$ & $\frac{1}{4}$ \\
2 & $\frac{1}{2}$ & 0 & $\frac{1}{2}$ \\
3 & 0 & $\frac{3}{4}$ & $\frac{1}{4}$
\end{tabular}

\begin{tabular}{l|ccc}
$\pi$ & $a$ & $b$ & $c$ \\
\hline 1 & $\frac{1}{2}$ & $\frac{1}{2}$ & 0 \\
2 & $\frac{1}{2}$ & 0 & $\frac{1}{2}$ \\
3 & 0 & $\frac{1}{2}$ & $\frac{1}{2}$
\end{tabular}

Fig. 2. The assignment $\pi$, which is sd-envy-free at $R$, sw-dominates $P S(R)$ at $R$, since $S p(\pi) \subsetneq S p\left(\pi^{p s}(R)\right)$.

Consider the $P S$ assignment $\pi^{p s}(R)$ and an object assignment, namely $\pi$, both of which are depicted above. Note that $\pi$ has a smaller support. Then, by Theorem $1, \pi \operatorname{sw}$-dominates $\pi^{p s}(R)$. Also, it is easy to check that $\pi$ is sd-envy-free, and any assignment that has a smaller support cannot be sd-envy-free.

\subsection{Sufficiency}

As the previous example shows, there are strict preference profiles for which there is an sdenvy-free assignment that sw-dominates the PS assignment. Then, when is it possible to have an sd-envy-free assignment that sw-dominates the $P S$ assignment? To answer this question, given $R \in \mathcal{R}^{S}$, we define a directed graph $G(R)$ as follows:

Definition. For each $R \in \mathcal{R}^{S}, G(R)$ is a directed graph where each agent-object pair is a vertex and for each vertex pair $(i, a),(j, b)$, there is an edge from $(i, a)$ to $(j, b)$, denoted by $(i, a) \rightarrow$ $(j, b)$, if for each pair of objects $x, y \in A$ such that $x R_{i} a$ with $\pi^{p s}(i, x)>0$ and $b P_{j} y$ with $\pi^{p s}(j, y)>0$, we have $x P_{j} y^{8}$

To paraphrase the definition for $(i, a) \rightarrow(j, b)$, let $U\left(R_{i}, a\right)$ denote the upper contour set of $R_{i}$ at $a$, that is, $U\left(R_{i}, a\right)=\left\{b \in A: b R_{i} a\right\}$. Let $U^{+}\left(R_{i}, a\right)$ denote the set of objects in

\footnotetext{
8 Note that if there exists no $x \in A$ such that $x R_{i} a$ and $\pi^{p s}(i, x)>0$, or if there exists no $y \in A$ such that $b P_{j} y$ and $\pi^{p s}(j, y)>0$, then, trivially, there is an edge from $(i, a)$ to $(j, b)$.
} 
$U\left(R_{i}, a\right)$ that are assigned to $i$ with positive probability at the $P S$ assignment. Then, $(i, a) \rightarrow$ $(j, b)$ if $U^{+}\left(R_{i}, a\right) \subset U\left(R_{j}, y\right)$ for each object $y$ such that $b P_{j} y$ with $\pi^{p s}(j, y)>0$. To put it more compactly, let $y^{j b}$ be the best object at $R_{j}$ such that $b P_{j} y$ with $\pi^{p s}(j, y)>0$. Then, we have $(i, a) \rightarrow(j, b)$ if $U^{+}\left(R_{i}, a\right) \subset U\left(R_{j}, y^{j b}\right)$. The following figure illustrates the $G(R)$ for Example 2. Note that if $(i, a) \rightarrow(j, b)$, then for each $y \in A$ with $b P_{j} y,(i, a) \rightarrow(j, y)$. The bold edges are the critical edges, in the sense that if $(i, a) \rightarrow(j, b)$, then there is no $z P_{j} b$ with $(i, a) \rightarrow(j, z)$. The dotted edges are the ones that are not critical.

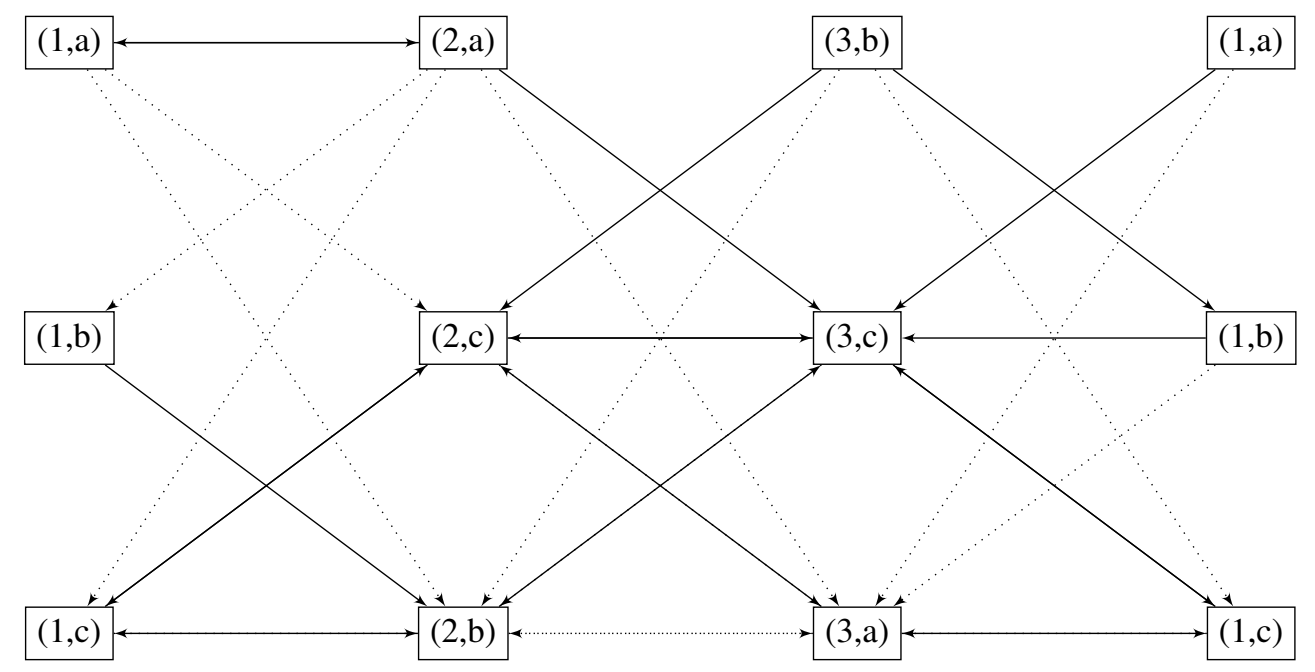

Roughly speaking $G(R)$ provides an ordinal account of "whose assignment is about to fail to be envy-free for whom and for which object at the $P S$ assignment." This interpretation of $G(R)$ follows from Lemma A.5 in which we show that for each $(i, a)$ and $(j, a)$ such that $a$ is assigned to $i$ and $j$ with positive probability, $(i, a) \rightarrow(j, a)$ implies that if we increase the probability that $a$ is assigned to $i$ in the $P S$ assignment, then the assignment of $j$ no longer stochastically dominates that of $i$. Moreover, $G(R)$ provides an ordinal account of these relationships, since configuration of $G(R)$ depends on whether an object is assigned to an agent or not in the $P S$ assignment, but it is independent of the particular assignment probabilities.

We observe that a special connectedness property of $G(R)$ plays a critical role in understanding when the $P S$ assignment is strongly sw-efficient among the sd-envy-free assignments. In graph theoretic language, a vertex $(i, a)$ is said to be connected to another vertex $(j, b)$ in $G(R)$ if there is a path, a sequence of vertices $v_{1}, v_{2}, \ldots, v_{k}$ such that $(i, a) \rightarrow v_{1} \rightarrow v_{2} \rightarrow \cdots v_{k} \rightarrow$ $(j, b)$. Next, we introduce the connectedness property that will be key for our results.

Definition. For each $R \in \mathcal{R}^{S}$ and $a \in A$, the graph $G(R)$ is $\boldsymbol{a}$-connected if for each $i, j \in N$ such that $\pi^{p s}(R)(i, a)>0,(i, a)$ is connected to $(j, a)$ in $G(R)$. The graph $G(R)$ is connected if it is $a$-connected for each $a \in A$.

Recall that we interpret the configuration of $G(R)$ as an account of "whose assignment is about to fail to be envy-free for whom and for which object at the $P S$ assignment". Now, suppose $G(R)$ fails to be $a$-connected for some object $a$. This means that we can alter the $P S$ assignment at $R$ without violating sd-envy-freeness. In this vein, our next result shows that if $G(R)$ is connected, then the $P S$ assignment is strongly sw-efficient among the sd-envy-free assignments. 
Before proceeding with the result, to get some familiarity with the connectedness notion, consider the following two extreme preference profiles. Suppose that in the first profile each agent has the same preference relation, whereas in the second profile each agent top-ranks a distinct object. The $P S$ assignment allocates each object equally between the agents at the first preference profile, and assigns each agent his top choice with probability one at the second preference profile. In both preference profiles, $G(R)$ is connected since for each $a \in A$ and $i, j \in N$ with $\pi^{p s}(i, a)>0$, we have $(i, a) \rightarrow(j, a)$. More specifically, for the first preference profile, where the preferences are exactly the same, observe that for each $a \in A$, if we restrict $G(R)$ to the vertex set $N \times\{a\}$ we obtain the complete graph. Similarly, for the second preference profile, for each $a \in A$, since there is a single agent $i \in N$ with $\pi(i, a)>0$, if we restrict $G(R)$ to the vertex set $N \times\{a\}$, then we obtain a star-shaped directed graph. Moreover, clearly at both preference profiles, the $P S$ assignment is the unique sd-efficient and sd-envy-free assignment. In fact, we next show that connectedness of $G(R)$ is sufficient for the $P S$ assignment to be strongly sw-efficient among the sd-envy-free assignments at $R$.

Proposition 1. For each $R \in \mathcal{R}^{S}$, if $G(R)$ is connected, then the PS assignment is strongly sw-efficient among the sd-envy-free assignments.

Proof. See Section A.2.

\subsubsection{Connected preference profiles}

For a given a preference profile $R$, we ask if the $P S$ assignment is strongly sw-efficient among the sd-envy-free assignments. The sufficient condition that we suggest in Proposition 1 is in terms of both the preference profile and the support of the $P S$ assignment. One can question if there is a family of preferences profiles such that at each member of this family, the associated $P S$ assignment is strongly sw-efficient among the sd-envy-free assignments, and can be identified without referring to the associated $P S$ assignment. In this section, we show that if a preference profile is connected in the vein of Sato (2013), then the desired conclusion holds. We present examples of connected preference profiles that are studied in the literature.

Sato (2013) introduces the notion of the connected domain, and shows that this is a necessary domain restriction for the equivalence of local and global strategy-proofness. First, we adopt the notion of a preference relation being adjacent to another from Sato (2013), and then introduce a preference profile being connected. Given a preference relation $R_{i}$, two objects $x$ and $y$ are consecutive in $R_{i}$ if $x$ and $y$ are ranked consecutively in $R_{i}$. Two preference relations $R_{i}$ and $R_{j}$ are adjacent if $R_{j}$ can be obtained by exchanging the positions of a unique pair of consecutive objects in $R_{i}$. Next, we define what is meant by a preference profile being connected.

Definition. For each $R \in \mathcal{R}^{S}$ and $i, j \in N$, there is a path in $R$ that connects $R_{i}$ to $R_{j}$ if there is a set of agents $\{1, \ldots k\} \subset N$ such that:

i. For each $l \in\{1, \ldots k-1\}, R_{l}$ is adjacent to $R_{l+1}$.

ii. $R_{i}$ is adjacent to $R_{1}$ and $R_{k}$ is adjacent to $R_{j}$.

A preference profile $R \in \mathcal{R}^{S}$ is connected if for each $i, j \in N$, there is a path in $R$ that connects $R_{i}$ to $R_{j}$. 
Connectedness of a preference profile can be interpreted as a richness condition, in that if two preferences appear in a profile then connectedness requires that a set of preferences, which roughly lies between these two, also appear at the same profile. Note that connectedness is a plausible condition especially when the market is large in the sense that it includes many agents but few objects. Next, we present some examples of connected preference profiles.

Example 3. A preference profile has full support (Liu and Pycia, 2016), if for each preference relation there is an agent in the society endowed with that preference relation. It is easy to see that if a preference profile has full support, then it is connected.

Example 4. Suppose that objects are indexed as $x_{1}, \ldots, x_{n}$ and consider preference relations that are single-peaked with respect to the indices of the objects. ${ }^{9}$ Now, for each $k \in\{1, \ldots, n\}$, let $\mathcal{R}_{k}$ be the collection of all preference relations that peaks at $x_{k}$. A preference profile $R$ is a rich single-peaked preference profile ${ }^{10}$ if there exist $k, m \in\{1, \ldots, n\}$ with $k<m$ such that there is an agent with preference $R_{i}$ at $R$ if and only if $R_{i} \in \cup_{\{k \leq l \leq m\}} \mathcal{R}_{l}$. It follows that if we form a preference profile $R$ by collecting all the single-peaked preferences that peaks at some $x_{l}$, where $k \leq l \leq m$ for some $k<m$, then $R$ is a rich single-peaked profile. To see that such preference profiles are connected, first note that for each $i \in\{1, \ldots, n\}, \mathcal{R}_{i}$ is connected. Next, for each $i \in\{1, \ldots, n-1\}$ and $R_{i} \in \mathcal{R}_{i}$, let $R_{i+1}$ be the preference relation with peak $x_{i+1}$ and the rest being same as $R_{i}$. Note that $R_{i+1} \in \mathcal{R}_{i+1}$ and is adjacent to $R_{i}$. Now, let $R_{i}$ and $R_{j}$ be two preferences that appear at $R$, it directly follows from our previous observations that $R_{i}$ is connected to $R_{j}$, hence $R$ is connected.

In our next result, we first show that if a preference profile $R$ is connected, then $G(R)$, the graph associated with $R$, is also connected. Then, the conclusion follows from Proposition 1.

Proposition 2. For each $R \in \mathcal{R}^{S}$, if $R$ is connected, then the PS assignment is strongly swefficient among the sd-envy-free assignments.

Proof. See Section A.3.

To see that there exists a preference profile $R$ that is not connected, but $G(R)$ is connected, consider a society with an even number of agents. Suppose that half of the society adopts the same preference relation $R_{1}$, whereas the other half adopts the opposite preference relation $R_{2}$, i.e. for each $a, b \in A, a R_{1} b$ if and only if $b R_{2} a$. It is easy to observe that such a preference profile $R$ is not connected, but $G(R)$ is connected.

\subsection{Necessity}

In this section, we first observe that for a given preference profile $R$, connectedness of $G(R)$ is not necessary for the $P S$ assignment to be strongly sw-efficient among the sd-envy-free as-

\footnotetext{
${ }^{9}$ See Black (1948) for the formal definition. Single-peaked preferences are one of the most commonly adopted class of preference relations due to their applicability and evasion from impossibilities.

10 One can similarly consider rich single-dipped preference profiles. See Barbera et al. (2012) for the definition of single-dipped preferences.
} 
signments. Next, we introduce the betweenness of a preference profile and show that under betweenness assumption, connectedness of $G(R)$ is a necessary condition.

Example 5. Let $N=\{1,2,3,4\}$ and $A=\{a, b, c, d\}$. Consider the following preference profile.

\begin{tabular}{cccc}
$R_{1}$ & $R_{2}$ & $R_{3}$ & $R_{4}$ \\
\hline$a$ & $a$ & $b$ & $c$ \\
$b$ & $c$ & $c$ & $a$ \\
$c$ & $d$ & $d$ & $d$ \\
$d$ & $b$ & $a$ & $b$
\end{tabular}

\begin{tabular}{|ccccc}
$\pi^{p s}(R)$ & $a$ & $b$ & $c$ & $d$ \\
\hline 1 & $\frac{1}{2}$ & $\frac{1}{4}$ & 0 & $\frac{1}{4}$ \\
2 & $\frac{1}{2}$ & 0 & $\frac{1}{4}$ & $\frac{1}{4}$ \\
3 & 0 & $\frac{3}{4}$ & 0 & $\frac{1}{4}$ \\
4 & 0 & 0 & $\frac{3}{4}$ & $\frac{1}{4}$
\end{tabular}

First note that objects $b$ and $c$ are exhausted simultaneously at time $3 / 4$. Since agent 1 ranks $c$ right below $b, R$ violates betweenness. Next, we argue that there is no path that connects the pair $(1, b)$ to $(3, b)$. To see this, first note that only $(4, c)$ is linked to $(3, b)$ and only $(2, c)$ and $(2, a)$ are linked to $(4, c)$. Similarly, note that only $(1, a)$ is linked to $(2, a)$ and $(2, c)$. Since $(1, b)$ is not linked to $(1, a),(2, c),(4, c)$ or $(3, b)$, there is no path that connects $(1, b)$ to $(3, b)$. Finally we argue that $\pi^{p s}(R)$ is the unique assignment that is sd-envy free and sd-efficient at $R$. To see this, first note that at any sd-envy-free and sd-efficient assignment at $R, a$ should be shared evenly between agents 1 and 2. Given this, to be sd-efficient 2 and 4 should eat from $c$. Now, for agent 4 not to envy agent 2, 4 should eat 3/4 of $c$. Since 1 and 3 rank $b$ over $d, 2$ and 4 should complete their assignments by equally eating from $d$. Thus assignments of agents 2 and 4 should be as in $\pi^{p s}(R)$. Next consider the assignment of agent 3. Since $a$ and $c$ are exhausted, 3 can eat from $b$ and $d$, let $p$ be the amount of $b$ that 3 eats. Now, note that the only value of $p$ that makes 1 and 3 not to envy each other is $3 / 4$. It follows that $\pi^{p s}(R)$ is the unique sd-efficient among sd-envy-free assignments. Thus we show that in the absence of betweenness, although $P S$ assignment is sw-efficient among sd-envy-free assignments, $G(R)$ may not be connected.

Next, we introduce a property which turns out to be key in understanding when connectedness is necessary for the $P S$ assignment to be strongly sw-efficient among the sd-envy-free assignments.

Definition. A preference profile $R \in \mathcal{R}^{S}$ satisfies betweenness if for each pair $a, b \in A$ that are simultaneously exhausted in the $P S$ algorithm at $R$ and for each $i \in N$ with $\pi^{p s}(i, a)>0$, there exists $c \in A$ such that $\pi^{p s}(i, c)>0$ and $a P_{i} c P_{i} b$.

To get some intuition for betweenness, first note that if a pair $a, b \in A$ are simultaneously exhausted in the $P S$ algorithm at $R$, and for some agent $i$, we have $\pi^{p s}(i, a)>0$, then this means $i$ prefers to eat $a$ instead of $b$. It follows that we necessarily have $a P_{i} b$. Betweenness additionally requires the existence of another object that is matched with $i$, and lies between $a$ and $b$ at $P_{i}{ }^{11}$

\footnotetext{
11 Note that the betweenness of a preference profile can be directly verified by only using the support of the $P S$ assignment. In section A.4, we show that betweenness implies the upper semi-continuity of $\operatorname{Sp}\left(\pi^{p s}(R)\right)$, a technical requirement which is critical for proving the result, but rather difficult to verify directly.
} 
Remark 1. A specific class of preference profiles that satisfy betweenness is the following. A preference profile $R$ satisfies distinct exhaustion condition if for each distinct pair of objects $a, b \in A, a$ and $b$ are exhausted at different times in the $P S$ algorithm. For example consider the preference profile at which each agent has the same preference relation over the objects. Then, since each agent-object pair is matched with positive probability in the $P S$ algorithm, the distinct exhaustion condition is directly satisfied. Since for such preference profiles, there is no object pair that are simultaneously exhausted, betweenness is directly satisfied. To see this, note that if a pair of objects $a$ and $b$ are simultaneously exhausted, then agents that exhaust $a$ can not get matched with $b .^{12}$

Our next result shows that if a preference profile $R$ satisfies betweenness, then connectedness of $G(R)$ is necessary for the $P S$ assignment to be strongly sw-efficient among the sd-envy-free assignments.

Proposition 3. For each $R \in \mathcal{R}^{S}$ that satisfy betweenness, if the PS assignment is strongly sw-efficient among the sd-envy-free assignments, then $G(R)$ is connected.

Proof. See Section A.4.

Once we identify when is it possible to sw-dominate the $P S$ assignment without sacrificing sd-envy-freeness, the next question is how to obtain such an assignment. The construction in the proof of Proposition 3 implicitly answers this question. Now, we revisit Example 2 to give a rough overview of how can we use this construction to obtain an sd-envy-free assignment that sw-dominates the $P S$ assignment. First, consider the preference profile $R$ and $\pi^{p s}(R)$. One can easily check that each object is exhausted at different times in $\pi^{p s}(R)$. Next, consider the graph $G(R)$. Note that if for each $x \in A$, we restrict the $G(R)$ to the vertex set $N \times\{x\}$, we obtain the three graphs below. It directly follows from their configuration that $G(R)$ is $a$-connected and $c$-connected. However, $G(R)$ is not $b$-connected, since $(1, b)$ is not connected to $(3, b)$. To see this, first note that neither $(1, b)$ nor $(2, b)$ is linked to $(3, b)$. Moreover, since only agent 3 top-ranks $b$ and is assigned to $c$ with positive probability, there is no $(i, x) \in N \times\{a, c\}$ with $(i, x) \rightarrow(3, b)$.
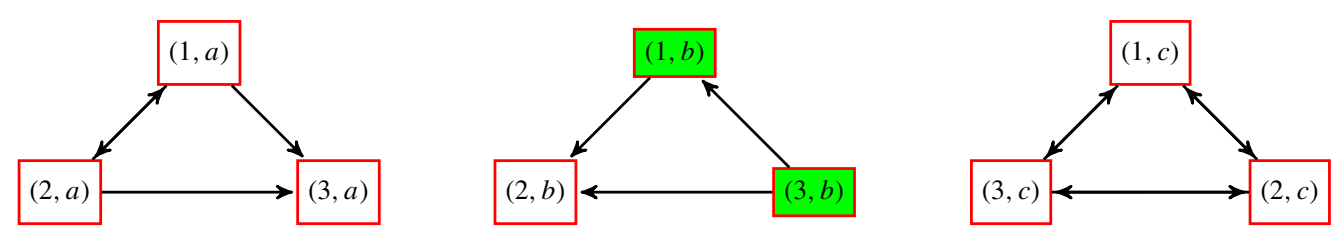

\footnotetext{
12 To see an example that satisfies betweenness but does not satisfy the distinct exhaustion condition, consider a society with an even number of (at least four agents) such that half of the society adopts the preference relation $R_{1}$ and the other half adopts the preference relation $R_{2}$. Suppose the only difference between $R_{1}$ and $R_{2}$ is that $a(b)$ is top-ranked at $R_{1}\left(R_{2}\right)$, whereas $b(a)$ is bottom-ranked at $R_{1}\left(R_{2}\right)$. Consider the $P S$ assignment for this society, since half of the society top ranks $a$ and the other half top ranks $b, a$ and $b$ are simultaneously exhausted. Therefore, the distinct exhaustion condition is not satisfied. To see that betweenness is satisfied, first note that only objects $a$ and $b$ are simultaneously exhausted. Since for those agents that eat $a(b), b(a)$ is bottom ranked, there exists another object that is matched with positive probability, and lies between $a$ and $b$.
} 
Now, since $(1, b)$ is not connected to $(3, b)$, we can transfer some amount of $b$ from 3 to 1 without violating sd-envy-freeness. Let us transfer the assignment of $b$ from 3 to 1 until any additional transfer makes 3 to envy 1 . This way we can transfer one-quarter the probability of $b$ from 3 to 1 . Hence agent 1's assignment is finalized, and we can add the $c$ share of agent 1 in $\pi^{p s}(R)$ to the $c$ assignment of agent 3. Thus, we obtain the assignment $\pi$ in Example 2, which is sd-envy-free and sw-dominates the $P S$ assignment.

Our results in this section are related to a strand of literature that aims to answer at which preference profiles $P S$ assignment is the unique sd-envy-free and sd-efficient assignment. ${ }^{13}$ It follows from our Proposition 3 and Corollary 1 that connectedness of $G(R)$ is sufficient for the $P S$ assignment being unique sd-envy-free and sd-efficient assignment among assignments which assign an agent-object pair a positive probability only if the $P S$ assignment assigns a positive probability to that pair. On the other hand, for arbitrary preference profiles, a necessary condition follows from the proof of our Proposition 3 in that if $P S$ assignment is the unique sd-efficient and sd-envy-free assignment at a preference profile $R$, then $G(R)$ must be connected. ${ }^{14}$

\section{Conclusion}

We propose the notion of social welfare efficiency and provide a clean ranking of sd-efficient assignments in terms of this new efficiency notion. This ranking enables us to compare wellknown assignment mechanisms such as the random serial dictatorship mechanism and the probabilistic serial mechanisms, which are incomparable according to sd-domination.

Our analysis in the first part of the paper shows that each sw-efficient assignment is essentially deterministic, indicating a trade-off between fairness and efficiency. It follows from this result that, in a setting where randomization is required to establish fairness, the best policy in terms of social welfare efficiency is to establish fairness with a minimum amount of randomization.

In the second part of the paper, we focus on the probabilistic serial mechanism. We question at which preference profiles the probabilistic serial assignment is sw-efficient among fair assignments. We show that a connectedness property of a directed graph induced by the preference profile provides a sufficient condition, which turns out to be also necessary if the given preference profile satisfies a betweenness condition.

\section{Appendix A}

\section{A.1. Proof of Theorem 1}

The following lemma, which easily follows from results in Manea (2008), plays a central role for the necessity part of our result.

Lemma A.1. For each $R \in \mathcal{R}$, if $\pi \in \Pi$ is sd-efficient at $R$, then there exist a utility profile $u$ consistent with $R$ and a function $v: A \rightarrow \mathbb{R}$ such that for each $(i, a) \in N \times A$,

\footnotetext{
13 Heo (2014) and Cho (2016) provide different sufficient conditions that are not necessary for the uniqueness of the $P S$ assignment.

14 In the proof of our Proposition 3 for each preference profile $R$ such that $G(R)$ is not connected, we construct an assignment that is sd-envy-free, sd-efficient and different from the $P S$ assignment. We use betweenness condition to additionally show that the constructed assignment weakly sd-dominates the $P S$ assignment.
} 
i. if $(i, a) \in \operatorname{Ext} \operatorname{Sp}(\pi, R)$, then $u_{i}(a)=v(a)$, and

ii. if $(i, a) \notin \operatorname{Ext} \operatorname{Sp}(\pi, R)$, then $u_{i}(a)<v(a)$.

Proof. For each $R \in \mathcal{R}$, suppose that $\pi \in \Pi$ is sd-efficient at $R$. The existence of $u$ and $v$ with the desired properties will easily follow from the way the utility profile is constructed in Manea (2008). For the sake of completeness, we first need to introduce the notation and the results that we need from Manea (2008). Consider the following binary relations on A:

i. $a \triangleright b$ iff there is $i \in N$ such that $a P_{i} b$ and $\pi_{i}(b)>0$.

ii. $a \bowtie b$ iff $a \not b$ and there is $i \in N$ such that $a I_{i} b$ and $\pi_{i}(b)>0$.

iii. $a \unrhd b$ iff $a \triangleright b$ or $a \bowtie b$.

iv. $a \triangleq b$ iff there is a sequence of objects $a_{1}, \ldots, a_{k}$ (with possibly repeated terms) such that $a_{1} \bowtie a_{2} \bowtie \ldots \bowtie a_{k} \bowtie a_{1}$ with $a, b \in\left\{a_{1}, \ldots, a_{k}\right\} .{ }^{15}$ Note that $\triangleq$ is an equivalence relation (reflexive, symmetric, transitive). For each $a \in A$, let $[a]$ denote the equivalence class of $a$.

v. $[a] \gg[b]$ iff $[a] \neq[b]$ and there are $a^{\prime} \in[a], b^{\prime} \in[b]$ such that $a^{\prime} \unrhd b^{\prime}$ (this relation is defined on the set of equivalence classes of $\triangleq$ ).

Since $\pi \in \Pi$ is sd-efficient at $R$, due to Manea (2008) there is a utility profile $u$ consistent with $R$ and a function $v: A \rightarrow \mathbb{R}$ with the following properties ${ }^{16}$ :

i. For each $a \in A, v(a)$ is the length of the longest chain of $\gg$ starting at $[a]$.

ii. For each $i \in A$ such that $\pi_{i}(a)>0$, we have $u_{i}(a)=v(a)$.

iii. For each $i \in A$ such that $\pi_{i}(a)=0$ and $\left\{b \in A \mid a R_{i} b, \pi_{i}(b)>0\right\}=\emptyset$, we have $u_{i}(a)<$ $\min _{b \in A} v(b)$.

iv. For each $i \in A$ such that $\pi_{i}(a)=0$ and $\left\{b \in A \mid a R_{i} b, \pi_{i}(b)>0\right\} \neq \emptyset$, we have $u_{i}(a)<$ $\max _{\left\{b \mid a R_{i} b, \pi_{i}(b)>0\right\}} v(b)+1$.

Now, we are ready to complete the proof. We will show that the utility profile $u$ and the function $v$ satisfying the above four properties also satisfies the two conditions in the statement of Lemma A.1. Clearly, for each $i \in N$ and $a \in A, u_{i}(a) \leq v(a)$. Therefore, we just need to show that $u_{i}(a)=v(a)$ iff $(i, a) \in \operatorname{Ext} \operatorname{Sp}(\pi, R)$.

Suppose that $(i, a) \in \operatorname{ExtSp}(\pi, R)$. Then, there are $\left(i_{1}, a_{1}\right), \ldots,\left(i_{k}, a_{k}\right) \in N \times A$ such that $(i, a) \sim_{(\pi, R)}\left(i_{1}, a_{1}\right) \sim_{(\pi, R)} \cdots \sim_{(\pi, R)}\left(i_{k}, a_{k}\right) \sim_{(\pi, R)}(i, a)$. Now, since $(i, a) \sim_{(\pi, R)}\left(i_{1}, a_{1}\right)$, we have $v(a) \geq u_{i}(a)=v\left(a_{1}\right)$. Similarly, for each $t \in\{2, \ldots, k-1\}, v\left(a_{t}\right) \geq u_{i_{t}}\left(a_{t}\right)=v\left(a_{t+1}\right)$, and $v\left(a_{k}\right) \geq u_{i_{k}}\left(a_{k}\right)=v(a)$. Thus, $u_{i}(a)=v(a)$.

Suppose that $u_{i}(a)=v(a)$. If $(i, a) \in \operatorname{Sp}(\pi)$, then obviously $(i, a) \in \operatorname{Ext} \operatorname{Sp}(\pi, R)$. So, suppose that $(i, a) \notin S p(\pi)$. Then, $\left\{b \in A \mid a R_{i} b, \pi_{i}(b)>0\right\} \neq \emptyset$, because otherwise $u_{i}(a)<$ $\min _{b \in A} v(b) \leq v(a)$. Let $b \in \arg \max _{b^{\prime} \in\left\{b \in A \mid a R_{i} b, \pi_{i}(b)>0\right\}} v\left(b^{\prime}\right)$. Note that $u_{i}(a)<v(b)+1$ and $a \unrhd b$. Then either $[a]=[b]$ or $[a] \gg[b]$. If $[a] \gg[b]$, then $v(a) \geq v(b)+1$, contradicting $v(a)=$ $u_{i}(a)<v(b)+1$. So, suppose that $[a]=[b]$. Then, there are $a_{1}, \ldots, a_{k}, a_{k+1}, \ldots, a_{K}$ such that $b \bowtie a_{1} \bowtie a_{2} \bowtie \ldots \bowtie a_{k} \bowtie a \bowtie a_{k+1} \bowtie a_{k+2} \bowtie \ldots \bowtie a_{K} \bowtie b$. Then, there is $i_{1} \in N$ such that $b I_{i_{1}} a_{1}, \pi_{i_{1}}\left(a_{1}\right)>0$; for each $t \in\{1, \ldots, k\}$, there is $a_{t}$ such that $a_{t-1} I_{i_{t}} a_{t}, \pi_{i_{t}}\left(a_{t}\right)>0$; and

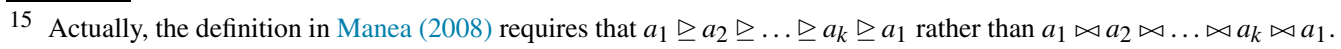
Given that $\pi$ is sd-efficient at $R$, the two definitions are equivalent (Katta and Sethuraman, 2006).

16 A result by Katta and Sethuraman (2006), which characterizes sd-efficient assignments in terms of a property of $\unrhd$, plays an important role in the analysis of Manea (2008). 
there is $i_{k+1}$ such that $a I_{i_{k+1}} a_{k}$. But then $\left(i_{1}, b\right) \sim_{(\pi, R)}\left(i_{2}, a_{1}\right) \sim_{(\pi, R)}\left(i_{3}, a_{2}\right) \sim_{(\pi, R)} \cdots \sim_{(\pi, R)}$ $\left(i_{k}, a_{k-1}\right) \sim_{(\pi, R)}\left(i_{k+1}, a_{k}\right) \sim_{(\pi, R)}(i, a) \sim_{(\pi, R)}\left(i_{1}, b\right)$. Thus, $(i, a) \in \operatorname{Ext} \operatorname{Sp}(\pi, R)$.

The following lemmas will be helpful to present a characterization of sw-domination.

Lemma A.2. For each $R \in \mathcal{R}$ if an assignment $\pi$ is sd-efficient at $R$, then there is a utility profile $u$ consistent with $R$ such that the following is true: an assignment $\pi^{\prime}$ maximizes $S W(u, \cdot)$ if and only if $\operatorname{Sp}\left(\pi^{\prime}\right) \subset \operatorname{Ext} \operatorname{Sp}(\pi, R)$.

Proof. A straightforward corollary to Lemma A.1.

Lemma A.3. For each $R \in \mathcal{R}$ and assignment $\pi$, there is an assignment $\pi^{*}$ such that $\operatorname{Sp}\left(\pi^{*}\right)=$ $\operatorname{ExtSp}(\pi, R)$ and for each utility profile u consistent with $R, S W\left(u, \pi^{*}\right)=S W(u, \pi)$.

Proof. Take any $\left(i_{0}, a_{0}\right) \in \operatorname{Ext} S p(\pi, R) \backslash S p(\pi)$. There exists $\left(i_{1}, a_{1}\right),\left(i_{2}, a_{2}\right), \ldots,\left(i_{k}, a_{k}\right) \in N \times$ $A$ such that $\left(i_{0}, a_{0}\right) \sim_{(\pi, R)}\left(i_{1}, a_{1}\right) \sim_{(\pi, R)} \ldots \sim_{(\pi, R)}\left(i_{k}, a_{k}\right) \sim_{(\pi, R)}\left(i_{0}, a_{0}\right)$. Starting from $\pi$, for some small enough $\epsilon>0$, by transferring $\epsilon$ probability of $a_{t}$ from $i_{t-1}$ to $i_{t}$ for each $t \in$ $\{1, \ldots, k\}$, and transferring $\epsilon$ probability of $a_{0}$ from $i_{k}$ to $i_{0}$, we can obtain an assignment $\pi^{\prime}$ such that $\pi_{i_{t}}^{\prime}\left(a_{t}\right)>0$ and $\pi_{i_{t}}^{\prime}\left(a_{t+1}\right)>0$ for each $t \in\{1, \ldots, k\}$, with the convention that $a_{k+1}=a_{0}$.

Note that $\operatorname{Sp}\left(\pi^{\prime}\right)=\operatorname{Sp}(\pi) \cup\left\{\left(i_{0}, a_{0}\right),\left(i_{1}, a_{1}\right),\left(i_{2}, a_{2}\right), \ldots,\left(i_{k}, a_{k}\right)\right\}$ and also $\operatorname{Sp}\left(\pi^{\prime}\right) \subset$ $\operatorname{Ext} \operatorname{Sp}(\pi, R)$. Moreover, each agent receives the same utility at $\pi^{\prime}$ and $\pi$. Hence, $S W\left(u, \pi^{\prime}\right)=$ $S W(u, \pi)$. Once we repeat this procedure for each $(i, a) \in \operatorname{Ext} \operatorname{Sp}(\pi, R) \backslash S p(\pi)$, we obtain the desired assignment $\pi^{*}$.

Lemma A.4. For each $R \in \mathcal{R}$ and assignment pair $\pi$ and $\pi^{\prime}$, if $\operatorname{Sp}(\pi) \subset S p\left(\pi^{\prime}\right)$, then for each utility profile u consistent with $R$, if $\pi^{\prime}$ is ex-ante efficient at $u$, then $\pi$ is ex-ante efficient at $u$.

Proof. Consider a decomposition of $\pi$ into deterministic assignments, say consisting of $\mu_{1}, \ldots, \mu_{k}$. First, we show that there is a decomposition of $\pi^{\prime}$ which includes $\mu_{1}, \ldots, \mu_{k}$. Since $S p(\pi) \subset S p\left(\pi^{\prime}\right)$, there is $\epsilon>0$ such that each entry of $z=\pi^{\prime}-\epsilon \pi$ is non-negative. Moreover, each row sum and each column sum of $z$ is $1-\epsilon$. Then, $\frac{1}{1-\epsilon} z \in \Pi$ and $\frac{1}{1-\epsilon} z$ can be written as a convex combination of deterministic assignments, say $\mu_{1}^{\prime}, \ldots, \mu_{t}^{\prime}$. Thus, $\pi^{\prime}=\epsilon \pi+z$ and therefore $\pi^{\prime}$ can be decomposed into $\mu_{1}, \ldots, \mu_{k}, \mu_{1}^{\prime}, \ldots, \mu_{t}^{\prime}$. Now, consider any utility profile $u$ consistent with $R$ at which $\pi^{\prime}$ is ex-ante efficient. Since $\pi^{\prime}$ maximizes $S W(u, \cdot)$, the sum of the utilities of the agents at each deterministic assignment in $\left\{\mu_{1}, \ldots, \mu_{k}, \mu_{1}^{\prime}, \ldots, \mu_{t}^{\prime}\right\}$ is the same and equal to $S W\left(u, \pi^{\prime}\right)$. Thus, $S W(u, \pi)=S W\left(u, \pi^{\prime}\right)$. Therefore, $\pi$ is ex-ante efficient whenever $\pi^{\prime}$ is ex-ante efficient.

Now, we are ready to prove Theorem 1.

If part: Suppose that $\pi^{\prime} \notin P^{s d}(R)$ and $\pi \in P^{s d}(R)$. Since $\pi^{\prime} \notin P^{s d}(R)$, there is no utility profile $u$ consistent with $R$ at which $\pi^{\prime}$ is ex-ante efficient. Since $\pi \in P^{s d}(R)$, by Lemma A.1 there is a utility profile $u$ consistent with $R$ at which $\pi$ is ex-ante efficient, implying that $\pi$ sw-dominates $\pi^{\prime}$.

Suppose that $\pi^{\prime} \in P^{s d}(R)$ and $\operatorname{Ext} \operatorname{Sp}(\pi, R) \subsetneq \operatorname{Ext} \operatorname{Sp}\left(\pi^{\prime}, R\right)$. By Lemma A.3, there is an assignment $\pi^{*}$ such that $\operatorname{Sp}\left(\pi^{*}\right)=\operatorname{Ext} \operatorname{Sp}\left(\pi^{\prime}, R\right)$ and for each utility profile $u$ consistent with $R$, $S W\left(u, \pi^{*}\right)=S W\left(u, \pi^{\prime}\right)$. Since $\operatorname{Sp}(\pi) \subset S p\left(\pi^{*}\right)$, it follows from Lemma A.4 that $\pi$ is ex-ante 
efficient whenever $\pi^{*}$ is ex-ante efficient. Since for each utility profile $u$ consistent with $R$, $S W\left(u, \pi^{*}\right)=S W\left(u, \pi^{\prime}\right)$, it follows that $\pi$ is ex-ante efficient whenever $\pi^{\prime}$ is ex-ante efficient.

Now, since $\pi \in P^{s d}(R)$, by Lemma A.1 there is a utility profile $u$ consistent with $R$ and a function $v: A \rightarrow \mathbb{R}$ satisfying the properties listed in Lemma A.1. Since $\operatorname{Ext} \operatorname{Sp}(\pi, R) \subsetneq$ $\operatorname{Ext} \operatorname{Sp}\left(\pi^{\prime}, R\right)$, there is a pair $(i, a) \in \operatorname{ExtSp}\left(\pi^{\prime}, R\right) \backslash \operatorname{ExtSp}(\pi, R)$. Moreover, $u_{i}(a)<v(a)$ and therefore $S W(u, \pi)>S W\left(u, \pi^{\prime}\right)$. Hence, $\pi$ sw-dominates $\pi^{\prime}$.

Only if part: Suppose that $\pi$ sw-dominates $\pi^{\prime}$ at $R$. Then, $\pi \in P^{s d}(R)$. If $\pi^{\prime} \notin P^{s d}(R)$, then we are done. So suppose that $\pi^{\prime} \in P^{s d}(R)$. Now, by Lemma A.2, there is a utility profile $u$ consistent with $R$ satisfying the property in Lemma A.2 for the assignment $\pi^{\prime}$. In particular, $\pi^{\prime}$ is ex-ante efficient at $u$. Since $\pi$ sw-dominates $\pi^{\prime}, \pi$ is also ex-ante efficient at $u$. Moreover, by Lemma A.3, there is an assignment $\pi^{*}$ such that $\operatorname{Sp}\left(\pi^{*}\right)=\operatorname{Ext} \operatorname{Sp}(\pi, R)$ and $S W\left(u, \pi^{*}\right)=S W\left(u, \pi^{\prime}\right)$. Thus, $\pi^{*}$ is also ex-ante efficient at $u$. By Lemma A.2, this is possible only if $\operatorname{Sp}\left(\pi^{*}\right) \subset \operatorname{Ext} \operatorname{Sp}\left(\pi^{\prime}, R\right)$. Therefore, we have $\operatorname{Ext} \operatorname{Sp}(\pi, R) \subset \operatorname{Ext} \operatorname{Sp}\left(\pi^{\prime}, R\right)$.

Next, suppose that $\operatorname{Ext} \operatorname{Sp}(\pi, R)=\operatorname{Ext} \operatorname{Sp}\left(\pi^{\prime}, R\right)$. Note that by Lemma A.3, there is an assignment $\pi^{*}$ such that $\operatorname{Sp}\left(\pi^{*}\right)=\operatorname{Ext} \operatorname{Sp}(\pi, R)$ and for each utility profile $u, S W\left(u, \pi^{*}\right)=S W(u, \pi)$. Similarly, there is an assignment $\pi^{* *}$ such that $\operatorname{Sp}\left(\pi^{* *}\right)=\operatorname{Ext} \operatorname{Sp}\left(\pi^{\prime}, R\right)$ and for each utility profile $u, S W\left(u, \pi^{* *}\right)=S W\left(u, \pi^{\prime}\right)$. But then, $S p\left(\pi^{*}\right)=S p\left(\pi^{* *}\right)$. Now, it follows from Lemma A.4 that for each utility profile $u, \pi^{*}$ is ex-ante efficient at $u$ iff $\pi^{* *}$ is ex-ante efficient at $u$. This leads a contradiction, since $\pi$ sw-dominates $\pi^{\prime}$ implies there is a utility profile at which $\pi$ and $\pi^{*}$ are ex-ante efficient but $\pi^{\prime}$ and $\pi^{* *}$ are not. Hence, we conclude that $\operatorname{Ext} \operatorname{Sp}(\pi, R) \subsetneq \operatorname{Ext} \operatorname{Sp}\left(\pi^{\prime}, R\right)$.

\section{A.2. Proof of Proposition 1}

First, we introduce some notation. For each $R \in \mathcal{R}, i \in N$, and $a \in A$, let $U\left(R_{i}, a\right)$ and $L\left(R_{i}, a\right)$ denote the upper and the lower contour sets of $R_{i}$ at $a$, that is, $U\left(R_{i}, a\right)=\{b \in A$ : $\left.b R_{i} a\right\}$ and $L\left(R_{i}, a\right)=\left\{b \in A: a R_{i} b\right\}$. Let $P_{i}$ stand for the strict part of the preference relation $R_{i}$. Let $U\left(P_{i}, a\right)$ and $L\left(P_{i}, a\right)$ denote the strict upper and the strict lower contour sets of $R_{i}$ at $a$, that is, $U\left(P_{i}, a\right)=\left\{b \in A: b P_{i} a\right\}$ and $L\left(P_{i}, a\right)=\left\{b \in A: a P_{i} b\right\}$. Let $V=N \times A$ denote the vertex set of $G(R)$ for each $R \in \mathcal{R}$.

Let $\pi$ be an sd-efficient probabilistic assignment at $R$. For a contradiction, suppose that $\pi$ is an sd-envy-free probabilistic assignment that weakly sw-dominates $\pi^{p s}$ at $R$, i.e. $\operatorname{Sp}(\pi) \subset S p\left(\pi^{p s}\right)$. We will show that, if $G(R)$ is connected, then $\pi=\pi^{p s}$, which will yield a contradiction. In showing that, the following result, which is Theorem 1 of Hashimoto et al. (2014), will be useful: $\pi=\pi^{p s}$ if and only if for each $a \in A$ and $i, j \in N$ such that $\pi(i, a)>0$, we have $\pi\left(j, U\left(R_{j}, a\right)\right) \geq \pi\left(i, U\left(R_{i}, a\right)\right)$. Next, we show that if $G(R)$ is connected, then for each $a \in A$ and $i, j \in N$ such that $\pi(i, a)>0$, we have $\pi\left(j, U\left(R_{j}, a\right)\right) \geq \pi\left(i, U\left(R_{i}, a\right)\right)$.

First, we show that for each $(i, a),(j, b) \in V$, if $(i, a) \rightarrow(j, b)$, then $\pi\left(j, U\left(P_{j}, b\right)\right) \geq$ $\pi\left(i, U\left(P_{i}, a\right)\right)$. Since $S p(\pi) \subset S p\left(\pi^{p s}\right)$, for each $x \in U\left(P_{i}, a\right)$ and each $y \in L\left(P_{j}, b\right)$ such that $\pi(i, x)>0$ and $\pi(j, y)>0$, we have $x P_{j} y$. Let $z$ be $R_{j}$-best object in $L\left(P_{j}, b\right)$ with $\pi(j, z)>0$. Since $(i, a) \rightarrow(j, b)$, for each $x \in U\left(R_{i}, a\right)$ with $(i, x) \in \operatorname{Sp}(\pi), x \in U\left(P_{j}, z\right)$. Since $\pi$ is envy free, $\pi\left(j, U\left(P_{j}, z\right)\right) \geq \pi\left(i, U\left(P_{j}, z\right)\right)$. Hence we obtain $\pi\left(j, U\left(R_{j}, b\right)\right) \geq$ $\pi\left(i, U\left(R_{i}, a\right)\right)$.

Now, since $G(R)$ is $a$-connected, there is a path that connects $(i, a)$ to $(j, a)$ in $G(R)$. From the above finding, it follows that $\pi\left(j, U\left(R_{j}, a\right)\right) \geq \pi\left(i, U\left(R_{i}, a\right)\right)$. 


\section{A.3. Proof of Proposition 2}

Let $R \in \mathcal{R}^{S}$ be connected. We show that for each $a \in A, G(R)$ is $a$-connected. Rest follows from Proposition 1. First we borrow some terminology from Sato (2013). For each pair of preference relations $R_{i}$ and $R_{j}$, if $R_{j}$ is obtained by exchanging the positions of the consecutive objects $x$ and $y$ in $R_{i}$ such that $x R_{i} y$, then we say that $y$ overtakes $x$ in moving from $R_{i}$ to $R_{j}$.

For each $a \in A$, to see that $G(R)$ is $a$-connected, consider any pair of distinct agents $i, j \in N$ such that $\pi^{p s}(i, a)>0$. We show that there is a path in $G(R)$ that connects $(i, a)$ to $(j, a)$.

Since $R$ is connected there is a path in $R$ that connects $R_{i}$ to $R_{j}$. That is, there is a set of agents with the associated preferences $\left\{R_{1}, \cdots, R_{k}\right\}$ at $R$ such that (i) and (ii) holds. Since $R_{i}$ is adjacent to $R_{1}$, there is a pair of objects $x, y \in A$ such that $y$ overtakes $x$ in moving from $R_{i}$ to $R_{1}$. Next, we show that there exists $a_{1} \in A$ such that $(i, a) \rightarrow\left(1, a_{1}\right), \pi^{p s}\left(1, a_{1}\right)>0$, and either $a$ and $a_{1}$ are exhausted simultaneously or $a_{1}$ is exhausted before $a$.

Case 1: If $a \notin\{x, y\}$, then we have either $x R_{i} a$ and $y R_{i} a$; or $a R_{i} x$ and $a R_{i} y$. For these two cases, since the position of $a$ does not change both at $R_{i}$ and $R_{1}$, it directly follows that $(i, a) \rightarrow(1, a)$. Moreover, in both cases, since $\pi^{p s}(i, a)=\pi^{p s}(1, a)$, we have $\pi^{p s}(1, a)>0$. Therefore, we can choose $a_{1}=a$.

Case 2: If $a \in\{x, y\}$, then this means for some $b \in A$, either (1) $a$ overtakes $b$ in moving from $R_{i}$ to $R_{1}$ or (2) $b$ overtakes $a$ in moving from $R_{i}$ to $R_{1}$.

Suppose (1) holds, that is $b R_{i} a, a R_{1} b$, and the rest of $R_{i}$ and $R_{1}$ are identical. For each $x^{\prime} \in A \backslash\{b\}$ with $x^{\prime} R_{i} a$ we have $x^{\prime} R_{1} a$. To conclude that $(i, a) \rightarrow(1, a)$, we have to show $\pi^{p s}(1, b)=0$. Since $\pi^{p s}(i, a)>0$ and $b R_{i} a$, it follows that $b$ is exhausted before $a$. Since $a R_{1} b$, and the rest of $R_{i}$ and $R_{1}$ are identical, it follows from the definition of PS mechanism that $\pi^{p s}(1, a)>0$ whenever $\pi^{p s}(i, a)>0$. Therefore $\pi^{p s}(1, a)>0$, and $\pi^{p s}(1, b)=0$ since $b$ was exhausted before $a$.

Suppose (2) holds, that is $a R_{i} b, b R_{1} a$, and the rest of $R_{i}$ and $R_{1}$ are identical. Since for each $x^{\prime} \in A$ with $x^{\prime} R_{i} a$ we have $x^{\prime} R_{1} a,(i, a) \rightarrow(1, a)$. If $\pi^{p s}(1, a)>0$, then we can choose $a_{1}$ as $a$. If $\pi^{p s}(1, a)=0$, then we show that $(i, a) \rightarrow(1, b)$ and $\pi^{p s}(1, b)>0$. Since $\pi^{p s}(1, a)=0$, it directly follows that $(i, a) \rightarrow(1, b)$. Since $b R_{1} a$ and the rest of $R_{i}$ and $R_{1}$ are identical, $\pi^{p s}(1, a)=0$ implies that 1 was eating $b$ while $i$ was eating $a$ and either $a$ and $b$ are exhausted simultaneously or $b$ is exhausted before $a$. Therefore, $\pi^{p s}(1, b)>0$ and we can choose $a_{1}=b$. Note that this is the only instance in which we choose $a_{1} \neq a$ and we know that either $a$ and $b$ are exhausted simultaneously or $b$ is exhausted before $a$.

By proceeding similarly, we can conclude that there is a set of objects $\left\{a_{1}, \ldots a_{k}, a_{j}\right\}$ such that $(i, a) \rightarrow\left(1, a_{1}\right) \cdots\left(k, a_{k}\right) \rightarrow\left(j, a_{j}\right)$. However, to show that $(i, a)$ is connected to $(j, a)$ in $G(R)$, we need a path that ends with $(j, a)$. Next, we argue that $\left(k, a_{k}\right) \rightarrow\left(j, a_{j}\right)$ implies $\left(k, a_{k}\right) \rightarrow(j, a)$. For this, it is sufficient to have $a_{j} R_{j} a$ whenever $a_{j} \neq a$. Note that it follows from our construction that either $a$ and $a_{j}$ are exhausted simultaneously or $a_{j}$ is exhausted before $a$. If $a_{j} \neq a$, then $a_{j}$ is exhausted before $a$. It follows that $a_{j} R_{j} a$, and $\left(k, a_{k}\right) \rightarrow\left(j, a_{j}\right)$ implies $\left(k, a_{k}\right) \rightarrow(j, a)$.

\section{A.4. Proof of Proposition 3}

To prove this result, first we show that for each $a \in A$ and $(i, a),(j, a) \in V$ such that $(i, a)$ is not linked to $(j, a)$ in $G(R)$, in the $P S$ assignment we can increase the probability that $i$ receives $a$ without causing $j$ to envy $i$. 
Lemma A.5. For each $a \in A$ and $(i, a),(j, a) \in V$ such that $\pi^{p s}(i, a)>0$ and $\pi^{p s}(j, a)>0$, if $(i, a) \nrightarrow(j, a)$, then there exists $\epsilon_{i j}>0$ such that $\pi^{p s}\left(j, U\left(R_{j}, a\right)\right)>\pi^{p s}\left(i, U\left(R_{j}, a\right)\right)+\epsilon_{i j}$.

Proof. For the proof we need the following two observations.

Observation A.1. Since $(i, a) \nrightarrow(j, a)$, there exists $x \in U\left(R_{i}, a\right)$ such that $\pi^{p s}(i, x)>0$ and at $R_{j}, j$ ranks $x$ below some $b \in L\left(R_{j}, a\right)$ such that $\pi^{p s}(j, b)>0$.

Observation A.2. For each $y \in U\left(P_{j}, a\right) \backslash U\left(R_{i}, a\right)$ such that $\pi^{p s}(j, y)>0$, we have $\pi^{p s}(i, y)=0$. Suppose not. Since $y \in L\left(P_{i}, a\right)$ and $\pi^{p s}(i, y)>0$, we have $\pi^{p s}\left(i, U\left(R_{i}, y\right)\right)>$ $\pi^{p s}\left(i, U\left(R_{i}, a\right)\right)$. By definition of $\pi^{p s}$, we have $\pi^{p s}\left(i, U\left(R_{i}, a\right)\right)=\pi^{p s}\left(j, U\left(R_{j}, a\right)\right)$. Then, $\pi^{p s}\left(i, U\left(R_{i}, y\right)\right)>\pi^{p s}\left(j, U\left(R_{j}, y\right)\right)$, which contradicts the definition of $\pi^{p s}$.

It directly follows from Observation A.1 and Observation A.2 that $\pi^{p s}\left(i, U\left(R_{i}, a\right)\right) \geq$ $\pi^{p s}\left(i, U\left(R_{j}, a\right)\right)+\pi_{i}^{p s}(x)$. Now, choose $\epsilon_{i j}=\frac{\pi_{i}^{p s}(x)}{2}$. Since by definition of $\pi^{p s}$ we have $\pi^{p s}\left(j, U\left(R_{j}, a\right)\right)=\pi^{p s}\left(i, U\left(R_{i}, a\right)\right)$, we obtain $\pi^{p s}\left(j, U\left(R_{j}, a\right)\right)>\pi^{p s}\left(i, U\left(R_{j}, a\right)\right)+\epsilon_{i j}$.

Next we introduce an auxiliary allocation mechanism, which is a generalization of the PS mechanism to a setup where the available capacity of an object is not necessarily 1 and can be an arbitrary amount. Let $q \in \mathbb{R}_{+}^{A}$ be a quota vector, which specifies, for each object, the available amount of the object. For a given $R \in \mathcal{R}^{S}$, agents eat starting from their most preferred objects at equal speeds as usual. The algorithm terminates when each object is exhausted (note that an agent may end up eating more than or less than 1 unit of objects). We denote the $P S$ assignment at $(R, q)$ by $\pi^{p s}(R, q)$. For each $q \in \mathbb{R}_{+}^{A}$, let $S(q)=\left\{a \in A: q_{a}>0\right\}$. As before, $S p(\pi(R, q))$ denotes the set of agent-object pairs that are assigned with positive probability at $\pi(R, q)$.

Definition. Let $R \in \mathcal{R}^{S}$ and $q \in \mathbb{R}_{+}^{A}$. We say that $\operatorname{Sp}\left(\pi^{p s}(R, \cdot)\right)$ is upper semi-continuous at $q \in \mathbb{R}_{+}^{A}$ if there exists an $\epsilon>0$ such that for each $q^{\prime} \in \mathbb{R}_{+}^{A}$ with $\left\|q^{\prime}-q\right\|<\epsilon$ and $S\left(q^{\prime}\right) \subset S(q)$, we have $\operatorname{Sp}\left(\pi^{p s}\left(R, q^{\prime}\right)\right) \subset S p\left(\pi^{p s}(R, q)\right)$.

Lemma A.6. Let $\overrightarrow{1}$ stand for the unit quota vector in which each object has a quota of 1 unit. For a given preference profile $R \in \mathcal{R}^{S}$, if $R$ satisfies betweenness, then $\operatorname{Sp}\left(\pi^{p s}(R, \cdot)\right)$ is upper semi-continuous at $\overrightarrow{1} \in \mathbb{R}_{+}^{A}$.

Proof. Consider $\pi^{p s}(R)$ (or equivalently $\pi^{p s}(R, \overrightarrow{1})$ ) and for each object $a \in A$, let $t\left(\pi^{p s}(R), a\right)$ be the set of objects that are exhausted before $a$. It follows from the definition of the PS mechanism that for each agent $i$ and object $a$, we have $\pi^{p s}(R)(i, a)>0$ if and only if $U\left(P_{i}, a\right) \subset$ $t\left(\pi^{p s}(R), a\right)$. Note that given the weak order of exhaustion times of the objects while running $P S$ at $R$ for different quota vectors $q$, one can identify the support of $\pi^{p s}(R, q)$. Therefore, given two quota vectors $q$ and $q^{\prime}$, if the order of exhaustion times of the objects are the same in $\pi^{p s}(R, q)$ and $\pi^{p s}\left(R, q^{\prime}\right)$, then $\operatorname{Sp}\left(\pi^{p s}(R, q)\right)=\operatorname{Sp}\left(\pi^{p s}\left(R, q^{\prime}\right)\right)$.

Now, let $\epsilon$ be such that for each $a, b \in A$ that are exhausted at different times in $\pi^{p s}(R)$, $0<\epsilon<\left|t_{a}-t_{b}\right| / n$. Note that for each $q^{\prime} \in \mathbb{R}_{+}^{A}$ with $\left\|q^{\prime}-\overrightarrow{1}\right\|<\epsilon$, none of the exhaustion orders will be reversed while obtaining $\pi^{p s}\left(R, q^{\prime}\right)$. That is, if an object $a$ is exhausted before another object $b$ at $\pi^{p s}(R)$, then $a$ is exhausted before $b$ at $\pi^{p s}\left(R, q^{\prime}\right)$ too. However, two objects that are exhausted simultaneously at $\pi^{p s}(R)$, may be exhausted at different times at $\pi^{p s}\left(R, q^{\prime}\right)$. For the rest, let $\pi$ stand for $\pi^{p s}(R)$ and $\pi^{\prime}$ for $\pi^{p s}\left(R, q^{\prime}\right)$. 
Now, by contradiction suppose that there exists a pair $(i, a) \in N \times A$ such that $\pi(i, a)=0$ but $\pi^{\prime}(i, a)>0$. It follows that $U\left(P_{i}, a\right) \not \subset t(\pi, a)$ but $U\left(P_{i}, a\right) \subset t\left(\pi^{\prime}, a\right)$. We obtain a contradiction by showing that there exists an object $c \in U\left(P_{i}, a\right) \backslash t\left(\pi^{\prime}, a\right)$. To see this, first recall that only the objects that are exhausted simultaneously at $\pi$ may be exhausted at different times at $\pi^{\prime}$. It follows that for each $x \in t\left(\pi^{\prime}, a\right) \backslash t(\pi, a), x$ is exhausted at the same time with $a$ at $\pi$. From among these let $b$ be the object that $i$ was eating when $a$ is exhausted at $\pi$. Since $a$ and $b$ are exhausted simultaneously at $\pi$, and $\pi(i, b)>0$, it follows from betweenness that there exists an object $c$ such that $\pi(i, c)>0$ and $b P_{i} c P_{i} a$. Now, since $a$ and $b$ are exhausted simultaneously at $\pi$ and $b P_{i} c, c$ is exhausted after $a$ at $\pi$. Therefore, by the choice of $q^{\prime}, c$ must be exhausted after $a$ at $\pi^{\prime}$. It follows that although $c \in U\left(P_{i}, a\right), c \notin t\left(\pi^{\prime}, a\right)$.

Lemma A.7. For a given preference profile $R \in \mathcal{R}^{S}$, let $\pi^{t}$ be the partial assignment that is obtained by running $P S$ until time $t \in[0,1]$ and for each $x \in A$, let $q_{x}^{t}=1-\pi^{t}(N, x)$. If $\operatorname{Sp}\left(\pi^{p s}(R, \cdot)\right)$ is upper semi-continuous at $\overrightarrow{1}$, then $\operatorname{Sp}\left(\pi^{p s}(R, \cdot)\right)$ is upper semi-continuous at $q^{t}$.

Proof. Let $\operatorname{Sp}\left(\pi^{p s}(R, \cdot)\right)$ be upper semi-continuous at $\overrightarrow{1}$. Then, there exists $\epsilon^{\prime}>0$ such that for each $q^{\prime} \in \mathbb{R}_{+}^{A}$ with $\left\|q^{\prime}-q\right\|<\epsilon^{\prime}$ and $S\left(q^{\prime}\right) \subset S(q)$, we have $\operatorname{Sp}\left(\pi^{p s}\left(R, q^{\prime}\right)\right) \subset$ $\operatorname{Sp}\left(\pi^{p s}(R, q)\right)$. Let $\epsilon$ be such that $0<\epsilon<\epsilon^{\prime}$. Note that for each $q^{\prime}$ with $\left\|q^{t}-q^{\prime}\right\|<\epsilon$ and $S\left(q^{\prime}\right) \subset S\left(q^{t}\right)$, we have $\|\left(\overrightarrow{1}-\left(\overrightarrow{1}-q^{t}+q^{\prime}\right) \|<\epsilon\right.$. Since $S p\left(\pi^{p s}(R)\right)$ is upper semi-continuous at $\overrightarrow{1}$, we obtain $\operatorname{Sp}\left(\pi^{p s}\left(R, \overrightarrow{1}-q^{t}+q^{\prime}\right)\right) \subset \operatorname{Sp}\left(\pi^{p s}(R)\right)$. Note that $\operatorname{Sp}\left(\pi^{p s}\left(R, \overrightarrow{1}-q^{t}+q^{\prime}\right)\right)=$ $S p\left(\pi^{p s}\left(R, q^{\prime}\right)\right) \cup S p\left(\pi^{t}\right)$ and $S p\left(\pi^{p s}(R)\right)=S p\left(\pi^{p s}\left(R, q^{t}\right)\right) \cup S p\left(\pi^{t}\right)$. Next, we argue that $\operatorname{Sp}\left(\pi^{p s}\left(R, q^{\prime}\right)\right) \subset S p\left(\pi^{p s}\left(R, q^{t}\right)\right)$. For this conclusion, it is sufficient to show that for each $(i, a) \in \operatorname{Sp}\left(\pi^{p s}\left(R, q^{\prime}\right)\right) \cap S p\left(\pi^{t}\right)$, we have $(i, a) \in \operatorname{Sp}\left(\pi^{p s}\left(R, q^{t}\right)\right)$. For each $(i, a) \in \operatorname{Sp}\left(\pi^{t}\right)$, if $(i, a) \in S p\left(\pi^{p s}\left(R, q^{\prime}\right)\right)$, then $q_{a}^{\prime}>0$. Since $S\left(q^{\prime}\right) \subset S\left(q^{t}\right)$, we have $q_{a}^{t}>0$. Thus we have $(i, a) \in \operatorname{Sp}\left(\pi^{t}\right)$ and $q_{a}^{t}>0$, note that this is possible only if $a$ is the object that is eaten by agent $i$ at time $t$. It follows that $i$ first eats $a$ at $\pi^{p s}\left(R, q^{t}\right)$, therefore $(i, a) \in \operatorname{Sp}\left(\pi^{p s}\left(R, q^{t}\right)\right)$.

Now, we are ready to complete the proof Proposition 2. By contradiction suppose there exists $a \in A$ such that $G(R)$ is not $a$-connected. First we observe that $a$ cannot be an object that is exhausted last in $\pi^{p s}(R)$. By contradiction, suppose there exists $k \in N$ such that $\pi^{p s}\left(k, U\left(R_{k}, a\right)\right)=1$. Now, we argue that $G(R)$ must be $a$-connected. To see this note that (1) for each $i \in N$ with $\pi^{p s}(i, a)>0, a$ is the last object that is assigned $i$ in $\pi^{p s}(R)$. (2) for each $j \in N$ with $\pi^{p s}(j, a)=0$, let $b$ be the last object that $j$ is assigned in $\pi^{p s}(R)$. Since both $a$ and $b$ are exhausted at last, we have $b R_{j} a$. It directly follows from (1) and (2) that for each $(i, a),(j, a) \in V$ such that $\pi(i, a)>0,(i, a) \rightarrow(j, a)$. In what follows we will construct an assignment $\pi \neq \pi^{p s}$ that is sd-envy-free, sd-efficient and $\operatorname{Sp}(\pi) \subset \operatorname{Sp}\left(\pi^{p s}\right)$.

First let us define a partial assignment $\pi^{\prime}: N \times A \rightarrow[0,1]$ such that for each $i \in N$ and $a \in A, \pi^{\prime}(i, A) \leq 1$ and $\pi^{\prime}(N, a) \leq 1$. Note that we can still consider sd-envy-freeness of $\pi^{\prime}$, and if $\pi^{\prime}$ is sd-envy-free then for each $i, j \in N$ we must have $\pi^{\prime}(i, A)=\pi^{\prime}(j, A)$. Now let $\pi^{\prime}$ be the partial assignment, which is obtained by running the $P S$ algorithm until $a$ is exhausted. That is, if $a$ is exhausted at time $t \leq 1$, then for each $i \in N$ we have (1) for each $b \in A$ such that $\pi^{p s}\left(i, U\left(R_{i}, b\right)\right) \leq t, \pi^{\prime}(i, b)=\pi^{p s}(i, b)$, (2) there is at most one object $c \in A$ such that $\pi^{\prime}(i, c)>0$ and $\pi^{p s}(i, c) \neq \pi^{\prime}(i, c),(3) \pi^{\prime}(i, A)=t$. Note that $\pi^{\prime}(N, a)=1$ and for each $b \in A$ that is exhausted after $a, \pi^{\prime}(N, b)<1$. Since $\pi^{\prime}$ is obtained through running the $P S$ algorithm, all the arguments for the envy-freeness of $\pi^{p s}$ holds for $\pi^{\prime}$. Thus, we conclude that $\pi^{\prime}$ is sdenvy-free. Next, by using the fact that $G(R)$ isn't $a$-connected, we will construct an sd-envy-free 
partial assignment $\pi^{\prime \prime}$ via making some small perturbations to $\pi^{\prime}$ on the assignment probabilities of the objects that are assigned with positive probability at $\pi^{\prime}$ just before time $t$.

Since $G(R)$ isn't $a$-connected, there exist $i^{*}, j^{*} \in N$ such that there is no path that connects $\left(i^{*}, a\right)$ to $\left(j^{*}, a\right)$. Let $I$ be the set of all $i \in N$ such that there is a path that connects $\left(i^{*}, a\right)$ to $(i, a)$ and let $J$ be the set of all $j \in N$ such that there is a path that connects $(j, a)$ to $\left(j^{*}, a\right)$. Note that since $\left(i^{*}, a\right) \rightarrow\left(i^{*}, a\right)$ and $\left(j^{*}, a\right) \rightarrow\left(j^{*}, a\right)$, we have $i^{*} \in I$ and $j^{*} \in J$. For each $i \in I$ and $j \in J$, since there is no path that connects $\left(i^{*}, a\right)$ to $\left(j^{*}, a\right)$, there cannot be any path that connects $(i, a)$ to $(j, a)$, so $(i, a) \nrightarrow(j, a)$. It follows from Lemma A.5 that for each $i, j \in N$ such that $\pi(i, a)>0$ and $(i, a) \nrightarrow(j, a)$, there exists $\epsilon_{i j}>0$ such that $\pi^{p s}\left(j, U\left(R_{j}, a\right)\right)>$ $\pi^{p s}\left(i, U\left(R_{j}, a\right)\right)+\epsilon_{i j}$. Now, for any $\epsilon \leq \min _{\{i, j \in N: \pi(i, a)>0 \text { and }(i, a) \nrightarrow(j, a)\}} \epsilon_{i j}$, let $2|I| \epsilon_{I}=$ $2|J| \epsilon_{J}=\epsilon$, so we have $\epsilon_{I}+\epsilon_{J} \leq \epsilon$. Next, we define $\pi^{\prime \prime}$ as follows:

i. For each $i \in I, \pi^{\prime \prime}(i, a)=\pi^{\prime}(i, a)+\epsilon_{I}$ and for any $b \neq a, \pi^{\prime \prime}(i, b)=\pi^{\prime}(i, b)$.

ii. For each $j \in J, \pi^{\prime \prime}(j, a)=\pi^{\prime}(j, a)-\epsilon_{J}$, let $b$ be the next consumable object for $j$ after $a$, then $\pi^{\prime \prime}(j, b)=\epsilon_{I}+\epsilon_{J}$ and finally for any $c$ except $a$ and $b, \pi^{\prime \prime}(j, c)=\pi^{\prime}(j, c)$.

iii. For each $k \notin I \cup J$ with $\pi(k, a)>0$, let $b$ be the next consumable object for $k$ after $a$. Now, let $\pi^{\prime \prime}(k, b)=\epsilon_{I}$ and for each $c \neq b, \pi^{\prime \prime}(k, c)=\pi^{\prime}(k, c)$.

iv. Finally for each $k \in N$ with $\pi(k, a)=0$, let $b$ be the lowest ranked object that is consumed with positive probability in $\pi^{\prime}$.

a. If $b$ is exhausted after $a$, then let $\pi^{\prime \prime}(k, b)=\pi^{\prime}(k, b)+\epsilon_{I}$ and for each $c \neq b, \pi^{\prime \prime}(k, c)=$ $\pi^{\prime}(k, c)$.

b. If $a$ and $b$ are exhausted at the same time, then let $c$ be the next object that is consumed by $k$ at $\pi^{p s}$. Let $\pi^{\prime \prime}(k, c)=\pi^{\prime}(k, c)+\epsilon_{I}$ and for each $d \neq c, \pi^{\prime \prime}(k, d)=\pi^{\prime}(k, d)$.

Now, we argue that $\pi^{\prime \prime}$ is envy-free. First, it is easy to see that by our choice of $\epsilon$ and Lemma A.5, no agent envies another because of $a$. Second, no agent envies another because of a previously exhausted object, since we kept the probabilities of all such objects as in $\pi^{P S}(R)$, which is envy-free. Finally no agent envies another because of his lowest-ranked object that he is assigned with positive probability, since for each agent the total probability that he is assigned to an object that is at least as good as that object is equal to $t+\epsilon_{I}$. To see this note that by construction of $\pi^{\prime \prime}$ for each $i \in N, \pi^{\prime \prime}(i, A)=\pi^{\prime}(i, A)+\epsilon_{I}=t+\epsilon_{I}$. Thus, we conclude that $\pi^{\prime \prime}$ is sd-envy-free. Next, note that while $\pi^{\prime \prime}(N, a)=1$, for some $b \neq a$ we might have $\pi^{\prime \prime}(N, b)>1$. Now, we argue that we can choose $\epsilon$ such that for each $b \in A, \pi^{\prime \prime}(N, b) \leq 1$. To see this, first observe that by construction of $\pi^{\prime \prime}$ for each $b$ that is exhausted after $a$ we have $\pi^{\prime \prime}(N, b) \leq \pi^{\prime}(N, b)+n \cdot \epsilon$. So, we can choose $\epsilon$ so small that $\pi^{\prime \prime}(N, b) \leq 1$. Hence, we obtain an sd-envy-free partial assignment $\pi^{\prime \prime}$ such that the assignment of $a$ is different from that of $\pi^{p s}(R)$.

Next, we extend the partial assignment $\pi^{\prime \prime}$ to an assignment $\pi^{*}$. Let $q, q^{\prime \prime} \in \mathbb{R}_{+}^{A}$ be the quota vectors of the objects such that for each $x \in A, q_{x}=1-\pi^{\prime}(N, x)$ and $q_{x}^{\prime \prime}=1-\pi^{\prime \prime}(N, x)$ respectively. Define the assignment $\pi^{*}=\pi^{\prime \prime}+\pi^{p s}\left(R, q^{\prime \prime}\right)$. First, we argue that $\operatorname{Sp}\left(\pi^{*}\right) \subset \operatorname{Sp}\left(\pi^{p s}(R)\right)$. To see this first note that, by the construction of $\pi^{\prime \prime}$, we have $\operatorname{Sp}\left(\pi^{\prime \prime}\right) \subset S p\left(\pi^{p s}(R)\right)$. Since $R$ satisfies betweenness, it follows from Lemma A.6 and A.7 that $\operatorname{Sp}\left(\pi^{p s}(R, \cdot)\right)$ is upper semicontinuous at $q$. Therefore we can choose $\epsilon$ so small that $\operatorname{Sp}\left(\pi^{p s}\left(R, q^{\prime \prime}\right)\right) \subset \operatorname{Sp}\left(\pi^{p s}(R, q)\right)$. Moreover, since at least $\pi^{*}\left(i^{*}, a\right) \neq \pi^{p s}\left(i^{*}, a\right), \pi^{*}$ is different from $\pi^{p s}(R)$. Since we can easily express $\pi^{*}$ as an eating mechanism, it is sd-efficient. Finally, we argue that $\pi^{*}$ is sd-envy-free. To see this, note that for each $i, j \in N$ and $x \in A$, we have $\pi^{*}\left(i, U\left(R_{i}, x\right)\right)=\pi^{\prime \prime}\left(i, U\left(R_{i}, x\right)\right)+$ $\pi^{p s}(R, q)\left(i, U\left(R_{i}, x\right)\right)$ and $\pi^{*}\left(j, U\left(R_{i}, x\right)\right)=\pi^{\prime \prime}\left(j, U\left(R_{i}, x\right)\right)+\pi^{p s}(R, q)\left(j, U\left(R_{i}, x\right)\right)$. Since 
$\pi^{\prime \prime}$ and $\pi^{p s}(R, q)$ are sd-envy-free, $\pi^{*}\left(i, U\left(R_{i}, x\right)\right) \geq \pi^{*}\left(j, U\left(R_{i}, x\right)\right)$. It follows that $\pi^{*}$ is sdenvy-free.

\section{References}

Barbera, S., Berga, D., Moreno, B., 2012. Domains, ranges and strategy-proofness: the case of single-dipped preferences. Soc. Choice Welf. 39, 335-352.

Birkhoff, G., 1946. Three observations on linear algebra. Rev. Univ. Nac. Tucumán, Rev. A 5, 147-151.

Black, D., 1948. On the rationale of group decision-making. J. Polit. Econ. 56, 23-34.

Bogomolnaia, A., Moulin, H., 2001. A new solution to the random assignment problem. J. Econ. Theory 100, $295-328$.

Cho, W.J., 2016. When is the probabilistic serial assignment uniquely efficient and envy-free? J. Math. Econ. 66, 14-25.

Dogan, B., Yildiz, K., 2016. Efficiency and stability of probabilistic assignments in marriage problems. Games Econ. Behav. 95 (1), 47-58.

Hashimoto, T., Hirata, D., Kesten, O., Kurino, M., Unver, M.U., 2014. Two axiomatic approaches to the probabilistic serial mechanism. Theor. Econ. 9 (1), 253-277.

Heo, E.J., 2014. The extended serial correspondence on a rich preference domain. Int. J. Game Theory 43, $439-454$.

Hylland, A., Zeckhauser, R., 1979. The efficient allocation of individuals to positions. J. Polit. Econ. 87 (2), $293-314$.

Katta, A.K., Sethuraman, J., 2006. A solution to the random assignment problem on the full preference domain. J. Econ. Theory 131, 231-250.

Liu, Q., Pycia, M., 2016. Ordinal efficiency, fairness, and incentives in large markets. Available at SSRN https://ssrn.com/ abstract $=1872713$.

Manea, M., 2008. A constructive proof of the ordinal efficiency welfare theorem. J. Econ. Theory 141, 276-281.

Manea, M., 2009. Asymptotic ordinal inefficiency of random serial dictatorship. Theor. Econ. 4, 165-197.

McLennan, A., 2002. Ordinal efficiency and the polyhedral separating hyperplane theorem. J. Econ. Theory 105, 435-449.

Pathak, P.A., 2008. Lotteries in student assignment: the equivalence of queueing and a market-based approach. Department of Economics, MIT. Unpublished paper.

Sato, S., 2013. A sufficient condition for the equivalence of strategy-proofness and nonmanipulability by preferences adjacent to the sincere one. J. Econ. Theory 148, 259-278.

Thomson, W., 2010. Strategy-proof allocation rules. University of Rochester. Mimeo.

Von Neumann, J., 1953. A certain zero-sum two-person game equivalent to the optimal assignment problem. In: Contributions to the Theory of Games, vol. 2. Princeton University Press, Princeton, New Jersey. 